\title{
Los determinantes de las variaciones en el rendimiento del oro
}

\author{
Determinants of changes in gold returns
}

\author{
Adán Díaz-Hernández ${ }^{*}$, José Carlos Ramírez Sánchez², Yuri Salazar Flores ${ }^{3}$ \\ 'Universidad Anáhuac México, México \\ Universidad Panamericana, México \\ ${ }^{3}$ Universidad Nacional Autónoma de México, México
}

Recibido el 20 de marzo de 2018; aceptado el 14 de septiembre de 2018

Disponible en Internet el: 9 de septiembre de 2019

\section{Resumen}

En este trabajo se caracteriza el comportamiento de los rendimientos logarítmicos del oro entre 1995 y 2017 con base en varios modelos de media y varianza condicionales, que incorporan efectos de asimetría y colas pesadas. Para tal efecto, se lleva a cabo, primero, un análisis basado en vectores autorregresivos con el fin de identificar los principales regresores externos y, luego, una serie de ajustes con distintas especificaciones del tipo AR-GARCH para pronosticar la volatilidad de las fluctuaciones del oro con base en dichos regresores. La conclusión principal es que esas fluctuaciones pueden ser adecuadamente explicadas por el comportamiento de las series de USDEER y SP500 de acuerdo con la especificación $\operatorname{AR}(1)-G A R C H(1,1)$ que tiene asociada una distribución t de Student. Esto quiere decir que los determinantes de largo plazo de las variaciones del rendimiento del oro están relacionados con las estrategias cambiarias y de protección contra el ciclo del mercado de valores por parte de los inversionistas.

Código JEL: C46, C51, D81

Palabras clave: Fluctuaciones del precio del oro; VAR; GARCH; Métricas de riesgo; Pronósticos de volatilidad

\footnotetext{
*Autor para correspondencia Correo electrónico adan.diaz@anahuac.mx (A. Díaz-Hernández).

La revisión por pares es responsabilidad de la Universidad Nacional Autónoma de México. 


\begin{abstract}
This paper explains the behavior of logarithmic gold returns between 1995 and 2017 by using several conditional mean and variance models that incorporate asymmetry and heavy tails effects. For this purpose, we conduct, first, an analysis based on standard autoregressive vectors in order to identify the main external regressors and, later, a sort of adjustments with different specifications of the ARGARCH type to forecast the volatility of gold-price fluctuations. The main conclusion is that these fluctuations can be adequately explained by the behavior of the USDEER and SP500 series according to the specification AR(1)-GARCH $(1,1)$ that has a Student $t$ distribution associated to it. This means that the long-term determinants of gold-return volatilities are related to exchange rate hedging strategies and anti-cyclical protection against stock markets variations by investors.
\end{abstract}

JEL code: C46, C51, D81

Keywords: Gold price fluctuations; VAR; GARCH; Risk measures; Volatility forecasts

\title{
Introducción
}

A raíz de la crisis financiera mundial de 2007-09 se ha suscitado un renovado interés por el estudio del comportamiento de los precios del oro. La razón obedece a las propiedades inherentes de este activo que, desde entonces, han permitido a los inversionistas administrar mejor sus riesgos mediante la construcción de portafolios con posiciones menos volátiles. Nos referimos a las propiedades de cobertura cambiaria e inflacionaria y a las que derivan de sus funciones como almacenador de valor, refugio seguro y diversificador de portafolios (Baur et al., 2016). Cada una de esas propiedades cobra especial importancia de acuerdo con las circunstancias generales que rodean a una economía. En épocas de crisis, por ejemplo, la marcada correlación negativa entre el oro y algunos activos financieros habilita a los inversionistas a usar el metal precioso, además de refugio seguro, como diversificador de portafolios (Trück y Liang, 2012). Del mismo modo, en tiempos de devaluaciones continuas, el oro es regularmente aprovechado como almacenador de valor en diferentes estrategias de cobertura cambiaria (Kristjanpoller y Minutolo, 2015).

Este documento pondera la importancia de esas propiedades en el largo plazo al estudiar los determinantes del precio del oro en una época signada por altas y bajas abruptas en la cotización internacional del metal precioso. En particular, su objetivo consiste en pronosticar la volatilidad en las variaciones de los rendimientos logarítmicos del oro expresado en dólares estadounidenses (USD), con base en el comportamiento de algunos factores macroeconómicos y financieros que tienen impactos diferenciados en el período comprendido entre Enero de 
1995 y Agosto de 2017. Para tal efecto, se realizan diversos análisis estadísticos sobre las series de rendimientos del oro que incluyen ajustes de modelos de media y varianza condicionales (tipo AR-GARCH) bajo distintos regímenes de riesgo. La ventana de observación sobre la que se hace el pronóstico de las volatilidades y su backtesting, representa una submuestra o conjunto de prueba conformada por los últimos 100 periodos mensuales disponibles en el periodo bajo estudio.

En el proceso de calibración estadística se toman aquellas extensiones comunes a la especificación estándar GARCH que mejor capturan (filtran) la dinámica de la volatilidad de las series, sin incurrir en sobre parametrización de los modelos. Para eso se hace un balance entre la precisión y el nivel de complejidad de las especificaciones GARCH, conforme a un riguroso análisis de validación de los modelos, así como de su pertinencia conceptual. Sobre este último punto cabe destacar, por ejemplo, que debido a que en las series de rendimientos mensuales del oro se identifican patrones autorregresivos (AR) en la media condicional, la metodología hace uso de vectores AR (VAR) para identificar el comportamiento de los factores o regresores externos de acuerdo con su contribución a la volatilidad (incertidumbre) en las fluctuaciones del rendimiento del oro. Este es una novedad del trabajo que está en línea con los reclamos de los autores que estudian los comovimientos del oro con otros factores bajo el enfoque de cópulas.

Las conclusiones del documento confirman algunos resultados ya probados en la literatura al consignar que los determinantes de la volatilidad de los rendimientos del oro entre 1995 y 2017 son de una importancia ampliamente reconocida, como son los casos de la tasa efectiva del dólar y de los índices accionarios (SP500). Pero también hay otros elementos nuevos que colocan al documento como pionero, al menos en el país, y que sugieren: que esos determinantes tienen una influencia más de largo que de corto plazo; que las especificaciones GARCH presentan un desempeño insuperable en el pronóstico de la volatilidad de los commodities (como el oro) no solo dentro sino, también, fuera de la muestra; y que la calibración de los modelos AR-GARCH es ideal para identificar la persistencia de los factores determinantes de las variaciones de los rendimientos del oro en series de datos divididas en múltiples horizontes y con problemas de asimetría y colas gordas.

El documento consta de dos secciones adicionales. La segunda ofrece el contexto general del problema al incluir una revisión somera del estado del arte, seguido de un examen exploratorio de las principales características de la información empleada (tamaño de muestra y justificación del periodo considerado) y de una descripción del comportamiento de las series de rendimientos del oro a través del tiempo. La tercera desarrolla el análisis de calibración de los modelos AR-GARCH bajo diferentes horizontes de riesgo, con el fin de encontrar el mejor predictor de pronósticos fuera de la muestra para el grupo de factores seleccionados. Las conclusiones discuten los principales resultados del documento. 


\section{El Contexto General}

\section{Los determinantes del precio (rendimiento) del oro en la literatura}

Los determinantes del precio del oro incluyen factores financieros y de la economía real. Entre ellos, la literatura destaca los precios de otros commodities (específicamente plata y petróleo), la tasa de interés, la inflación, el tipo de cambio, la volatilidad del mercado de valores, el índice de precios al consumidor, las tasas rezagadas del PIB industrial y de los agregados monetarios (como el M2), las noticias sobre expectativas de crecimiento y los shocks macroeconómicos (Poshakwale y Mandal, 2016; Pierdzioch et al., 2016). La variabilidad de cada uno de estos factores, así como sus intrincadas interrelaciones dificultan la identificación de los determinantes de las fluctuaciones del precio (rendimiento) del oro, sobre todo, porque sus dinámicas son estado-dependientes y, por tanto, no es posible establecer asociaciones definitivas entre ellos a lo largo del tiempo. Los comovimientos del oro con los factores macroeconómicos o financieros cambian radicalmente con los periodos de estrés del mercado y eso puede incrementar o disminuir la importancia de algunas de sus propiedades (Piplack y Straetmans, 2010). Poshakwale y Mandal (2016) señalan que si esos comovimientos se intensifican (atenúan) en épocas de contracción económica es muy probable que la propiedad de refugio seguro del oro se vea comprometida (potenciada).

Ante este panorama, los estudios han adoptado dos caminos complementarios para encontrar los determinantes en el cambio del precio (rendimiento) del oro de acuerdo con metodologías de pronóstico bien diferenciadas al interior (pronóstico no predictivo) o fuera de la muestra (pronóstico predictivo). El primero es la identificación de los factores causantes de la volatilidad de las fluctuaciones del precio (o rendimiento) del oro con la ayuda de diferentes modelos de la familia GARCH o alguna versión mixta de éstos con ciertas heurísticas, como son los métodos de aprendizaje supervisado, redes neuronales o algoritmos de clasificadores. El segundo camino es la caracterización de las estructuras de dependencia de los comovimientos del oro y otros factores, como los arriba mencionados, mediante el uso de cópulas con regímenes cambiantes.

Los resultados principales en ambos casos sostienen que: a) no hay consenso sobre la preminencia de algunos factores sobre otros en la explicación de la volatilidad de las fluctuaciones del precio (rendimiento) del oro (Pierdzioch et al., 2016). Algunos estudios encuentran que las variaciones del dólar afectan más a los rendimientos del oro que el resto de los factores macroeconómicos (Tully y Lucey, 2007), mientras que otros sostienen que estos últimos son los únicos importantes cuando se usan datos intra-diarios (Cai et al., 2001); b) los efectos de los factores son muy inestables cuando se implementan pronósticos dentro de la muestra, debido a la existencia de elementos idiosincráticos en el precio de los commodities (Pierdzioch et al., 2016; Batten et al., 2010; Vivian and Wohar, 2012); c) los modelos que presentan mejores 
ajustes varían, en términos generales, de acuerdo con los tipos de pronósticos y datos considerados. Para series de tiempo de baja frecuencia, las especificaciones TARCH o IGARCH resultan excelentes predictores del comportamiento del precio del oro dentro de la muestra (véase, por ejemplo, Trück y Liang, 2012); muy al contrario de lo que sucede cuando los datos son intra-diarios o los pronósticos son fuera de la muestra, ya que en ese caso la introducción de los modelos GARCH en la capa oculta de una red neuronal, por ejemplo, puede ofrecer mejores resultados (Kristjanpoller y Minutolo, 2015); y d) a diferencia de los análisis tradicionales de series de tiempo, los modelos de cópulas revelan que: los comovimientos del oro son altamente dependientes del régimen bajo estudio y no solo de un periodo concreto (es decir dependen de la presencia o no de inflación o de las diferencias entre fases de expansión o contracción económicas, por mencionar unos casos); la importancia de un determinante es función directa de su contribución a la incertidumbre de un grupo de variables; y las propiedades del oro no son univocas en el sentido de que si bien el metal precioso resulta un buen ejemplo de cobertura para los bienes raíces en época de inflación puede no serlo en épocas de alzas en las tasas de interés (Poshakwale y Mandal, 2016).

\section{Selección del periodo de estudio y tamaño de muestra}

Una característica sobresaliente de los estudios anteriores es la ausencia de una distinción clara entre los determinantes de corto y largo plazo. No hay en las investigaciones mencionadas ningún tratamiento estadístico orientado a establecer las diferencias en el peso de los factores macroeconómicos y financieros, arriba listados, por duración de períodos. Tampoco se encuentra referencia explícita alguna a las propiedades permanentes o temporales del oro que están asociadas a dichos factores. El periodo seleccionado y el análisis estadístico de la sección III están dirigidos, precisamente, a atender este vacío en la literatura al buscar identificar los factores de largo plazo y, por ende las propiedades permanentes del oro, que determinan las variaciones en su rendimiento. Con esa idea en mente, consideramos todos los tramos de la figura 1, más adelante explicada, y no sólo sus periodos ascendentes o descendentes como es la práctica regular en la literatura. Obviamente, al proceder de esta manera esperamos detectar a los determinantes de largo plazo que sobreviven a las tendencias alcistas y bajistas del oro.

La base de datos aquí utilizada es obtenida de la plataforma Bloomberg LT (https://www. bloomberg.com/) y contiene información histórica de los precios de cierre diarios (5879 observaciones) y mensuales (272 observaciones) para la onza de oro puro expresada en USD (OROSpot). El periodo de observación arranca en un año de estabilidad con niveles bajos en los precios internacionales del oro (Enero de 1995) y termina en Agosto de 2017 un vez que el mercado entra, de nueva cuenta, en una segunda fase de estabilidad pero ahora con niveles altos en sus precios. Es decir, seleccionamos un periodo suficientemente largo para incluir 
toda clase de tendencias y niveles en el medio y los extremos. Asimismo, la periodización permite incluir series básicas para el estudio de los determinantes, como es la tasa de cambio efectiva del dólar (USDEER), que de otra manera no podrían ser contempladas adecuadamente. Como se sabe, la serie de este índice, en particular, incluye divisas de economías emergentes como el peso mexicano (MXN), cuya información se encuentra disponible a partir de 1994, justamente el año en que el MXN adopta el esquema de libre flotación.

\section{Las características de la información sobre los rendimientos del oro}

La Figura 1 muestra las series de tiempo de precios y rendimientos logarítmicos calculados a escalas diaria y mensual para todo el periodo de observación. ${ }^{1}$ En ambas escalas se aprecian
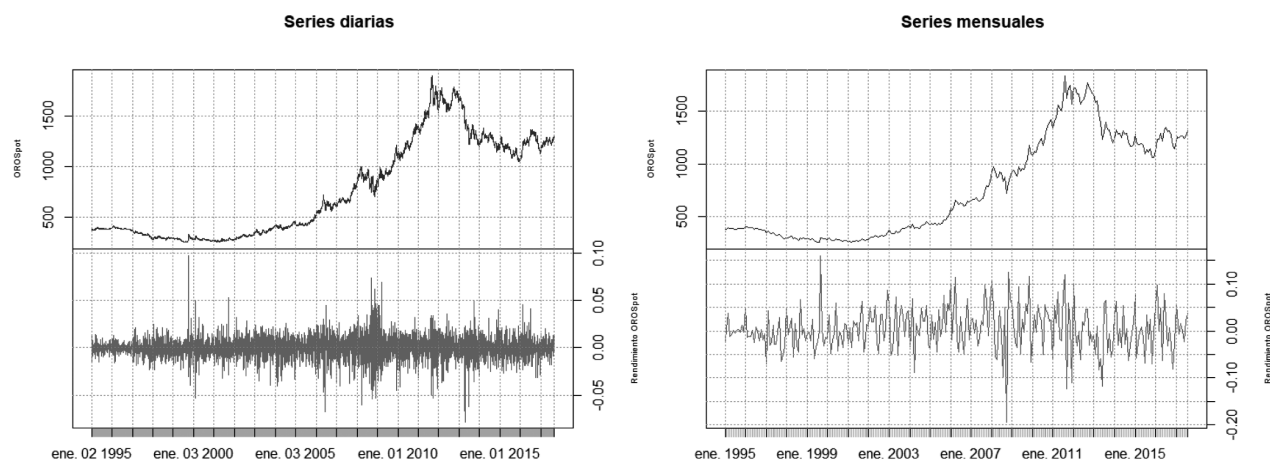

Figura 1. Series de tiempo del precio (azul) y de rendimientos (rojo) de la onza de oro en dólares americanos (OROSpot) para datos diarios (panel izquierdo) y mensuales (panel derecho). Fuente: elaboración propia con datos de Bloomberg LT (1995-2017).

cambios en la tendencia y en los niveles de volatilidad a lo largo del tiempo (intermitencia). ${ }^{2}$ Sobre las tendencias destacan dos épocas muy definidas: una ascendente entre 2002 y 2011 y otra descendente a partir de 2012 (ver las líneas azules de la figura 1). Durante la primera época, los precios del oro observan un sostenido crecimiento después de la caída de los índices accionarios, como el NASDAQ, y la quiebra de empresas tecnológicas (telecom crash) entre 2000 y 2002. El episodio conocido como la burbuja DotCom (por su vínculo con las empresas de la tecnología de la información) interrumpe un periodo de relativa estabilidad en la década de los años noventa, en el que el oro es visto como almacenador de valor, para

\footnotetext{
${ }^{1}$ Los rendimientos son calculados sobre bases diarias $(d)$ y mensuales $(m)$ empleando la expresión $R_{t}=\ln \left(P_{t} / P_{t-1}\right)$ para $t=d$ y $t=m$, donde $P_{t}$ denota el precio de cierre (diario o mensual, según corresponda).

${ }^{2}$ Las volatilidades realizadas (VR) mensuales se estiman como $R V_{t}=\frac{1}{D_{t}} \sum_{i=1}^{D_{t}} R_{t, i}^{2}$ donde $t=m$, en tanto que $D_{t}$ indica los números de días del mes $t$ y $R_{t, 1}, \ldots, R_{t, D_{t}}$ denota la muestra de rendimientos diarios observados en el mes $t$; y las anualizadas como $\sigma_{t}^{2}=12 R V_{t}$.
} 
inaugurar otro propio de un mercado alcista (bull market) en el que el metal se convierte en refugio seguro y elemento activo en la diversificación de portafolios. En esta época se aprecian tres picos sobresalientes en las series diarias, dos pequeños y uno grande, que se explican por el inicio de la guerra de Irak en 2003, la crisis inmobiliaria en 2008, el inicio de la crisis europea en 2009 y su consecuente impacto en el mercado de valores en Septiembre de 2011. La segunda época comprende los años entre 2012 y 2015 y se caracteriza por experimentar una caída sostenida en la cotización del oro, como consecuencia del "sobrecalentamiento" del mercado del oro iniciado en septiembre de 2012 (Pierdzioch et al.,2016).

En correspondencia con estas tendencias, las líneas rojas de la Figura 1 muestran un patrón que exhibe cúmulos o periodos alternados de altas y bajas volatilidades entre 1999 y 2000 (con un pico predominante a la alza en 1999) 2008 y 2010 (con un pico predominante a la baja en 2008) y 2011 y 2013 (con picos simétricos a la alza y a la baja). Este patrón produce rendimientos sobre el OROSpot muy dispares a lo largo del tiempo. De acuerdo con la Figura 2, el comportamiento mensual de los rendimientos acumulados del OroSpot sobre distintos horizontes presenta fuertes movimientos en ganancias y pérdidas, sobre todo a partir de 2005. En concreto la máxima ganancia anualizada ocurre durante 2006, en tanto que la máxima pérdida se presenta en 2013. Otros episodios de grandes pérdidas se registran entre 1997 y 1998 con las crisis asiática y rusa, así como de grandes ganancias entre 2008 y 2012 en plena crisis financiera mundial.

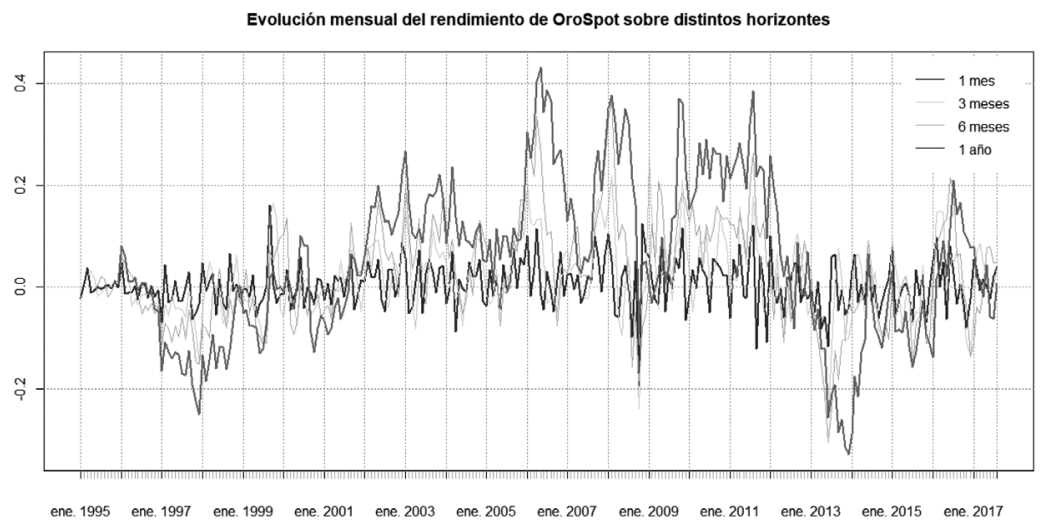

Figura 2. Cálculo mensual del rendimiento del OROSpot acumulado sobre distintos horizontes de riesgo (ventanas móviles) mensuales (azul), trimestrales (verde), semestrales (naranja) y anuales (rojo). Fuente: elaboración propia con datos de Bloomberg LT (1995-2017).

La Tabla 1 presenta las principales estadísticas descriptivas de los rendimientos logarítmicos de OROSpot para frecuencias diarias, mensuales, trimestrales y anuales ( $\sin$ traslape), así como el cálculo mensual de rendimientos acumulados trimestrales y anuales con ventanas móviles. Ahí 
se puede observar que los valores medios y medianos de los rendimientos son positivamente más grandes a medida que ampliamos la ventana de observación de días a meses o a años. Es decir, que entre más largo es el horizonte de inversión más alto son los rendimientos medios o medianos del oro. Pero, a diferencia de otros estudios, estos valores son significativamente menores debido a la amplitud del periodo considerado. Para series que incluyen solo la tendencia ascendente de la figura 1, la media de los rendimientos diarios es hasta 2000 veces más grandes que la registrada en nuestra muestra (0.04 vs 0.0002), por lo que no siempre son comparables estos resultados con los de la literatura existente (compárese, por ejemplo, estos valores con los de Trück y Liang (2012), quienes consideran una muestra entre enero de 1999 y diciembre de 2008). En concreto podemos asegurar que las estadísticas de los rendimientos del periodo ascendente en la cotización del oro son equivalentes a los valores anualizados de la Tabla 1, con lo que, entre más pequeña es la ventana de observación de la Tabla, más altos son los coeficientes de variación, más leptocurticas son las series de rendimientos y menos normales las distribuciones de rendimientos (los valores p reportados en el último renglón de la Tabla 1 para la estadística de prueba Jarque-Bera (JB) indican que esas distribuciones no son normales para frecuencias diarias, mensuales y trimestrales, al 5\% de significancia).

Tabla 1

Estadísticas descriptivas para los rendimientos del OROSpot bajo distintas frecuencias

\begin{tabular}{lcccccc}
\hline & \multicolumn{3}{c}{ Rendimientos (no traslapados) } & \multicolumn{2}{c}{ Cálculo mensual (móvil) } \\
\hline Media & Diario & Mensual & Trimestral & Anual & Trimestral & Anual \\
Mediana & 0.0002 & 0.0045 & 0.0136 & 0.0537 & 0.0135 & 0.0543 \\
DesvESt & 0.0002 & 0.0012 & 0.0115 & 0.0539 & 0.0047 & 0.0496 \\
Sesgo & 0.0104 & 0.0464 & 0.0680 & 0.1522 & 0.0733 & 0.1541 \\
Curtosis & -0.0319 & -0.0361 & -0.6706 & -0.6930 & 0.1363 & 0.0892 \\
Mínimo & 9.4668 & 4.1580 & 4.5413 & 3.0697 & 3.8897 & 2.5344 \\
Máximo & -0.0780 & -0.1931 & -0.2577 & -0.3301 & -0.2577 & -0.3301 \\
MaxDesv & 0.0977 & 0.1604 & 0.1487 & 0.2704 & 0.2511 & 0.4313 \\
JB & 9.4418 & 4.1617 & 3.7899 & 2.1686 & 3.5183 & 2.7983 \\
\hline
\end{tabular}

Fuente: elaboración propia.

\section{Los determinantes de las fluctuaciones de los rendimientos del oro}

\section{Un modelo VAR estándar}

Con la finalidad de desarrollar una metodología que caracterice las variaciones del OROSpot sobre distintos horizontes de riesgo, incluimos las siguientes variables económicas (reales) 
y financieras que han sido ampliamente destacadas en la literatura: tasa de cambio efectiva del dólar (USDEER), precio del barril de petróleo West Texas Intermediate en USD (WTI), índice Standard\&Poor's 500 (SP500), índice de precios al consumidor de EUA (USCPI), tasa de interés interbancaria LIBOR de USD (LibUSD), tasa de desempleo de EUA (USunEmp) e índice de producción industrial de EUA (USIProd). La elección de cada variable obedece a las propiedades del oro que queremos investigar. Con USDEER se busca evaluar la propiedad del oro como elemento decisivo de cobertura cambiaria; con WTI se investiga su rol como diversificador de portafolios que incluyen otros commodities; con SP500 se mide la calidad del oro como refugio seguro contra las volatilidades del mercado de valores; con USCPI se explora su papel en la cobertura contra la inflación y, finalmente, con los factores LibUSD, USunEMp y USIProd se pretende analizar su función contra las volatilidades del ciclo de negocios.

Debido a que no hay en la literatura un modelo establecido para el pronóstico de la volatilidad del precio (rendimiento) del oro, estas variables son indistintamente usadas como predictores exógenos, en los modelos de series de tiempo, o como variables de control, en los modelos heurísticos o de cópulas (Baur et al., 2016). Nosotros seguiremos la práctica habitual adoptada por los estudios de series de tiempo de considerar a los factores arriba mencionados como regresores externos en la explicación de las variaciones de los rendimientos del oro, sin atender a su naturaleza estado-dependiente (sobre este punto volveremos en las conclusiones). En este sentido, se espera que los regresores tengan el signo correspondiente a la activación de la propiedad del oro; esto es: un signo positivo para los rezagos del OROSpot (estacionalidad), USDEER (cobertura cambiaria), USCPI (cobertura inflacionaria), USunEmp (cobertura contra el ciclo económico) y LIBUSD (cobertura contra el diferencial de tasas) y un negativo para WTI (diversificación de portafolios que incluyen otros commodities), USIProd (cobertura contra inversiones en el ciclo industrial) y SP500 (cobertura contra el ciclo del mercado de valores).

$\mathrm{El}$ primer paso para determinar el efecto temporal que tienen estos regresores sobre el OROSpot consiste en analizar su comportamiento en el periodo de referencia. La figura 3 muestra que la mayoría de las series observan componentes de efectos de constante y tendencia cuando se consideran los niveles de cada factor. Para verificar la existencia de raíz unitaria y evaluar el orden de integración y estacionariedad de las series se aplican las pruebas de Dickey-Fuller aumentada (ADF) sobre niveles y primeras diferencias y las de Kwiatkowski-Phillips-Schmidt-Shin (KPSS) y Phillips-Perron (PP) (ver Tabla 2). En el caso de las variables OROSpot, USDEER, WTI y SP500 se toman los precios logarítmicos, de tal suerte que sus primeras diferencias correspondan a los rendimientos. El análisis considera componentes deterministas de constante y tendencia en el tiempo, así como el efecto de términos explicativos dados por los rezagos de la variable. Para un nivel de significancia del $5 \%$ se concluye que, con excepción de USunEmp y USIProd (cuya prueba KPSS indica que la afirmación es válida al 1\% de significancia), todas las series son integradas de orden 1 . 
El segundo paso es seleccionar los factores o regresores mediante el proceso VAR(p) expresado como:

$$
\boldsymbol{y}_{t}=A_{1} \boldsymbol{y}_{t-1}+\cdots+A_{p} \boldsymbol{y}_{t-p}+\boldsymbol{u}_{t}
$$

donde $\boldsymbol{y}_{t}=\left(y_{1 t}, \ldots, y_{k t}\right)^{\prime}$ es el conjunto de eventuales regresores, $A_{i}(k \times k), \quad i=1, \ldots, p$, las matrices que contienen los coeficientes sujetos a estimación y $\boldsymbol{u}_{t}$ el vector de errores k-dimensional que satisface las condiciones de que $E\left(\boldsymbol{u}_{t}\right)=\mathbf{0}$ y la matriz $E\left(\boldsymbol{u}_{t} \boldsymbol{u}_{t}^{\prime}\right)=\boldsymbol{\Sigma}_{\boldsymbol{u}}$ sea definida positiva e invariante en el tiempo (Véase Sims (1980); Hamilton (1994) y Johansen (1995), entre otros).

La Tabla 3 contiene las estimaciones de los coeficientes de la ecuación para el OROSpot de acuerdo con diferentes modelos del tipo (1) compuestos por regresores y términos de constante y tendencia, que son seleccionados mediante un proceso de eliminación secuencial. Los modelos parten del más general, en el que se incorporan todos los regresores mencionados, junto con su constante y tendencia, y concluyen con la especificación más particular que contiene a los factores sobrevivientes con valores $p<0.05$. Cada modelo tiene asociado al pie de la Tabla las pruebas de Portmanteau y ARCH-LM, con las que se confirma o rechaza, respectivamente, las hipótesis nulas de no autocorrelación en los residuales y de inexistencia de efectos ARCH.
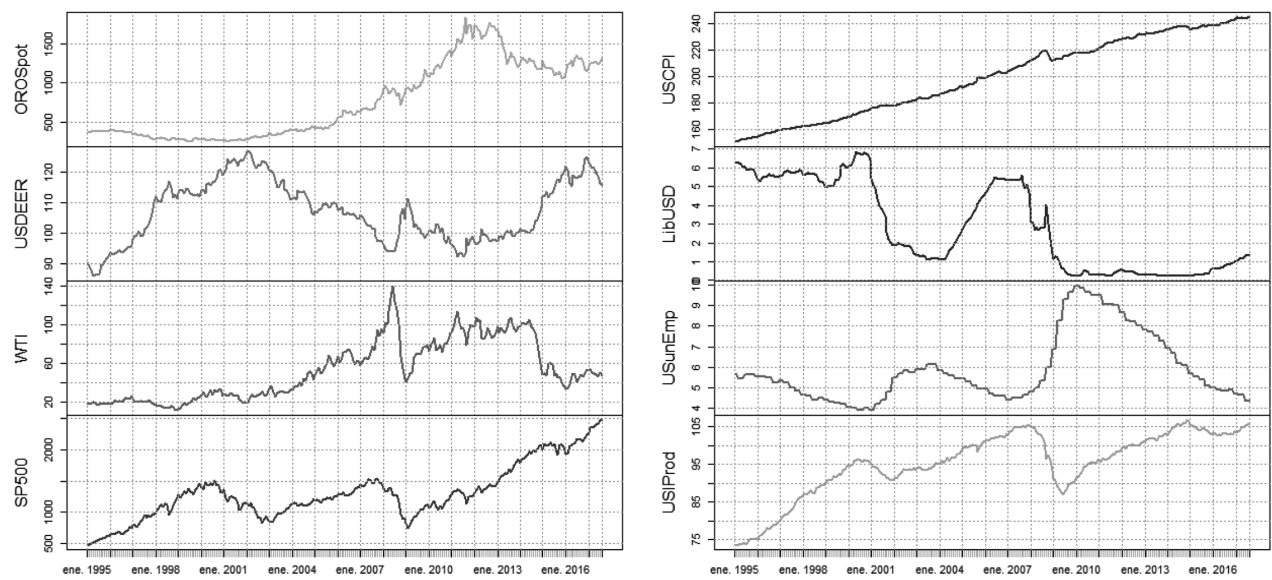

Figura 3. Series de tiempo mensuales de los indicadores globales considerados. Fuente: elaboración propia con datos de Bloomberg LT (1995-2017). 
Tabla 2

Valores de la prueba ADF para las series en niveles (con componentes deterministas de constante y tendencia y dos rezagos) y en primeras diferencias (con componente determinista constante y un rezago)

\begin{tabular}{|c|c|c|c|c|c|c|c|c|}
\hline \multirow{2}{*}{ Variable } & \multirow{2}{*}{$\begin{array}{l}\text { Componentes } \\
\text { deterministas }\end{array}$} & \multirow{2}{*}{ Rezagos } & \multirow{2}{*}{$\begin{array}{c}\text { ADF } \\
\text { calculada }\end{array}$} & \multicolumn{3}{|c|}{ Valores críticos } & \multirow{2}{*}{ KPSS } & \multirow{2}{*}{$\begin{array}{c}\text { Calculated } \\
\text { PP }\end{array}$} \\
\hline & & & & $1 \%$ & $5 \%$ & $10 \%$ & & \\
\hline $\ln ($ OROSpot $)$ & Constante, tendencia & 2 & -1.73 & -3.98 & -3.42 & -3.13 & 1.08 & -1.69 \\
\hline$\Delta \ln ($ OROSpot $)$ & Constante & 1 & -12.90 & -3.44 & -2.87 & -2.57 & 0.27 & -18.13 \\
\hline USDEER & Constante, tendencia & 2 & -2.08 & -3.98 & -3.42 & -3.13 & 0.98 & -1.80 \\
\hline$\triangle U S D E E R$ & Constante & 1 & -10.84 & -3.44 & -2.87 & -2.57 & 0.23 & -14.74 \\
\hline$W T I$ & Constante, tendencia & 2 & -1.75 & -3.98 & -3.42 & -3.13 & 1.22 & -1.65 \\
\hline$\Delta W T I$ & Constante & 1 & -10.84 & -3.44 & -2.87 & -2.57 & 0.14 & -14.48 \\
\hline$S P$ & Constante, tendencia & 2 & -2.25 & -3.98 & -3.42 & -3.13 & 0.65 & -2.36 \\
\hline$\triangle S P$ & Constante & 1 & -11.52 & -3.44 & -2.87 & -2.57 & 0.20 & -15.09 \\
\hline USCPI & Constante, tendencia & 2 & -1.80 & -3.98 & -3.42 & -3.13 & 1.53 & -0.77 \\
\hline$\triangle U S C P I$ & Constante & 1 & -10.83 & -3.44 & -2.87 & -2.57 & 0.40 & -10.82 \\
\hline LibUSD & Constante, tendencia & 2 & -1.70 & -3.98 & -3.42 & -3.13 & 0.45 & -0.80 \\
\hline$\triangle L i b U S D$ & Constante & 1 & -8.86 & -3.44 & -2.87 & -2.57 & 0.30 & -9.83 \\
\hline USunEmp & Constante, tendencia & 2 & -0.11 & -3.98 & -3.42 & -3.13 & 0.71 & -0.09 \\
\hline$\triangle U$ SunEmp & Constante & 1 & -11.56 & -3.44 & -2.87 & -2.57 & 0.54 & -16.39 \\
\hline USIProd & Constante, tendencia & 2 & -2.34 & -3.98 & -3.42 & -3.13 & 1.05 & -2.22 \\
\hline AUSIProd & Constante & 1 & -7.91 & -3.44 & -2.87 & -2.57 & 0.68 & -13.02 \\
\hline
\end{tabular}

Nota: Los valores críticos asociados a la estadística de prueba KPSS para las series en niveles son: 0.22, 0.15, 0.12 (con niveles de significancia del 1\%,5\% y 10\%, respectivamente); y los valores correspondientes para las series en diferencias son: $0.74,0.46,0.35$. En el caso de la prueba $\mathrm{PP}, \mathrm{los}$ valores críticos asociados a las estadísticas calculadas son: $-4.00,-3.43,-3.14$ (series en niveles) y $-3.46,-2.87$ y -2.57 (series en diferencias).

Fuente: elaboración propia

Tabla 3

Estimaciones de los parámetros asociados a la ecuación de OROSpot de los modelos VAR calibrados durante el proceso de eliminación secuencial

\begin{tabular}{lccccccc}
\hline Parámetros & Modelo 1 & Modelo 2 & Modelo 3 & Modelo 4 & Modelo 5 & Modelo 6 & Modelo 7 \\
\hline rezago1 OROSpot & 0.8623 & 0.8633 & 0.8619 & 0.8865 & 0.9586 & 0.9803 & 0.9883 \\
& $(4.3 \mathrm{E}-55)$ & $(2.1 \mathrm{E}-71)$ & $(2.7 \mathrm{E}-72)$ & $(1.3 \mathrm{E}-91)$ & $(9.6 \mathrm{E}-199)$ & $(3.8 \mathrm{E}-220)$ & $(2.7 \mathrm{E}-256)$ \\
rezago1 USDEER & -0.2250 & -0.2243 & -0.1829 & -0.1444 & 0.0901 & 0.0555 & 0.0646 \\
& $(1.69 \mathrm{E}-02)$ & $(1.81 \mathrm{E}-02)$ & $(4.36 \mathrm{E}-02)$ & $(4.70 \mathrm{E}-02)$ & $(4.67 \mathrm{E}-03)$ & $(3.96 \mathrm{E}-02)$ & $(8.50 \mathrm{E}-03)$ \\
rezago WTI & -0.0358 & -0.0348 & -0.0148 & & & & \\
& $(8.45 \mathrm{E}-02)$ & $(6.79 \mathrm{E}-02)$ & $(1.87 \mathrm{E}-01)$ & & & & -0.0355 \\
rezago SP500 & -0.0838 & -0.0839 & -0.0635 & -0.0623 & -0.0458 & -0.0248 & $(2.58 \mathrm{E}-02)$ \\
& $(4.46 \mathrm{E}-04)$ & $(4.87 \mathrm{E}-04)$ & $(4.74 \mathrm{E}-03)$ & $(6.46 \mathrm{E}-03)$ & $(4.42 \mathrm{E}-02)$ & $(2.04 \mathrm{E}-01)$ & \\
rezago USCPI & 0.0179 & & & & & &
\end{tabular}


http://dx.doi.org/10.22201/fca.24488410e.2018.1973

\begin{tabular}{|c|c|c|c|c|c|c|c|}
\hline \multirow[t]{2}{*}{ rezago LibUSD } & 1.3006 & 1.2996 & 1.2095 & 1.0206 & 0.6887 & 0.2964 & \\
\hline & $(6.64 \mathrm{E}-04)$ & $(5.55 \mathrm{E}-04)$ & $(1.79 \mathrm{E}-03)$ & $(6.34 \mathrm{E}-03)$ & $(6.68 \mathrm{E}-02)$ & $(3.28 \mathrm{E}-01)$ & \\
\hline \multirow[t]{2}{*}{ rezago USunEmp } & 1.7426 & 1.7306 & 1.4224 & 1.1281 & 0.7149 & & \\
\hline & $(5.49 \mathrm{E}-04)$ & $(4.33 \mathrm{E}-04)$ & $(1.58 \mathrm{E}-03)$ & $(2.29 \mathrm{E}-03)$ & $(5.47 \mathrm{E}-02)$ & & \\
\hline \multirow[t]{2}{*}{ rezago USIProd } & 0.2043 & 0.2035 & & & & & \\
\hline & $(1.60 \mathrm{E}-01)$ & $(1.79 \mathrm{E}-01)$ & & & & & \\
\hline \multirow[t]{2}{*}{ constante C } & 2.0985 & 2.1150 & 1.9263 & 0.6401 & & & \\
\hline & $(2.58 \mathrm{E}-03)$ & $(4.97 \mathrm{E}-04)$ & $(1.43 \mathrm{E}-03)$ & $(1.86 \mathrm{E}-01)$ & & & \\
\hline \multirow[t]{2}{*}{$\begin{array}{l}\text { tendencia } \mathrm{T} \\
\text { (tiempo) }\end{array}$} & 0.0015 & 0.0015 & 0.0015 & 0.0012 & 0.0006 & 0.0002 & 0.0002 \\
\hline & $(1.95 \mathrm{E}-01)$ & $(1.98 \mathrm{E}-05)$ & $(2.36 \mathrm{E}-05)$ & $(2.42 \mathrm{E}-05)$ & $(4.07 \mathrm{E}-04)$ & $(4.09 \mathrm{E}-03)$ & $(3.31 \mathrm{E}-03)$ \\
\hline \multirow[t]{2}{*}{ Portmanteau } & 2287.70 & 1948.04 & 1197.38 & 1197.38 & 1200.11 & 804.25 & 241.55 \\
\hline & $(2.00 \mathrm{E}-16)$ & $(2.00 \mathrm{E}-16)$ & $(2.00 \mathrm{E}-16)$ & $(2.00 \mathrm{E}-16)$ & $(2.00 \mathrm{E}-16)$ & $(2.00 \mathrm{E}-16)$ & $(5.01 \mathrm{E}-02)$ \\
\hline \multirow[t]{2}{*}{$\begin{array}{l}\text { ARCH-LM } \\
\text { univariada }\end{array}$} & 11.25 & 11.46 & 8.42 & 8.42 & 11.25 & 12.36 & 11.04 \\
\hline & $(3.38 \mathrm{E}-01)$ & $(3.23 \mathrm{E}-01)$ & $(5.88 \mathrm{E}-01)$ & $(5.88 \mathrm{E}-01)$ & (3.38E-01) & $(2.62 \mathrm{E}-01)$ & $(3.54 \mathrm{E}-01)$ \\
\hline
\end{tabular}

Nota: Los valores $\mathrm{p}$ se presentan entre paréntesis y en negritas se indican los casos en que la variable se elimina por registrar un valor $p>0.05$.

Fuente: elaboración propia.

Tabla 4

Estimaciones de los parámetros asociados a la ecuación de OROSpot en el modelo VAR ajustado (Modelo 7)

\begin{tabular}{lcccc}
\hline \multicolumn{1}{c}{ Parámetros } & Estimación & Error estándar & Estadística t & Valor $\mathrm{p}$ \\
\hline rezago1 OROSpot & 0.9883 & 0.0068 & 145.4868 & $2.69 \mathrm{E}-256$ \\
rezago1 USDEER & 0.0646 & 0.0244 & 2.6513 & 0.0085 \\
rezago SP500 & -0.0355 & 0.0158 & -2.2412 & 0.0258 \\
tendencia T (tiempo) & 0.0002 & 0.0001 & 2.9643 & 0.0033 \\
\hline
\end{tabular}

Nota: Las estimaciones de los coeficientes, errores estándar, estadísticas t y valores p son robustas ante la presencia de heterocedasticidad y autocorrelación.

Fuente: elaboración propia.

La Tabla 4 presenta los resultados finales del modelo VAR ajustado en los que se deja de manifiesto que los estimadores de los coeficientes resultan robustos a heterocedasticidad y autocorrelación serial (HAC), según la prueba de Newey and West (1987), y que los residuales no exhiben efectos ARCH. Cabe mencionar que se usa HAC porque la prueba de Portmanteau en el modelo ajustado 7 ofrece información poco convincente al no encontrar evidencia para rechazar la hipótesis nula de no autocorrelación serial. La Tabla A1 del Apéndice resume las ecuaciones del modelo VAR ajustado. 
Con el fin de detectar posibles anomalías en los procesos de error asociados a cada sistema de ecuaciones de los modelos VAR, la Tabla 5 muestra diferentes pruebas multivariadas así como su correspondiente valor $\mathrm{p}$ entre paréntesis. De las tres primeras columnas se desprende que los vectores de residuales no exhiben correlación serial (prueba Edgerton-Shukur), ni siguen una distribución normal (Prueba de Jarque-Bera), aunque presentan efectos ARCH en un contexto multivariado sobre cada uno de los regresores considerados (prueba ARCH-LM multivariada). Esto último quiere decir que, contrario a lo que sucede con el caso univariado, las primeras diferencias de los vectores residuales tienen efectos de heteroscedasticidad que requieren un tratamiento especial mediante la calibración de modelos GARCH, como veremos enseguida.

Tabla 5

Pruebas de validación sobre los residuales de las especificaciones VAR ajustadas

\begin{tabular}{cccc}
\hline \multicolumn{4}{c}{ Estadísticas de pruebas multivariadas } \\
\hline Edgerton-Shukur & Jarque-Bera & ARCH-LM & Causalidad \\
1.155 & 70.417 & 459.87 & {$[0.026]$} \\
$(0.2135)$ & $(3.36 \mathrm{E}-13)$ & $(0.0003)$ & $<1.72 \mathrm{E}-11>$ \\
\hline
\end{tabular}

Nota: Los valores p aparecen entre paréntesis.

Fuente: elaboración propia.

Un aspecto importante de la misma Tabla 5 es la prueba de la cuarta columna con la que se busca validar los efectos de causalidad de los regresores o variables causas del modelo 7 (USDEER y SP500) sobre el OROSpot o variable efecto. Para tal caso se consideran las siguientes dos hipótesis nulas: a) que el conjunto de regresores no causa en el sentido de Granger (1969) a la variable efecto; y b) que existe correlación cero entre la variable efecto y los procesos de error asociados a las variables causas, cuyo valor p está entre $<>$ de acuerdo con una prueba tipo Wald denominada de causalidad instantánea (Lütkepohl, 2006). En ambas situaciones se rechazan las hipótesis nulas y, por tanto, es posible asegurar que existe causalidad en el sentido de Granger entre las variables.

Con la identificación de los factores y la existencia de su causalidad resulta importante hacer dos anotaciones que fundamentan la robustez de los resultados. La primera es que, en la implementación de la última prueba, así como en la matriz de varianza-covarianza de las especificaciones VAR, se utilizan estimadores HAC y HC de White (1980) para asegurar la significancia estadística de los coeficientes, respectivamente. Su uso es inevitable pues, de lo contrario, se corre el grave riesgo de hacer una incorrecta evaluación de los determinantes, como sucede con el caso de WTI cuyo coeficiente resulta significativo bajo la estimación de Mínimos Cuadrados Ordinarios (MCO) estándar en el modelo 7 de la Tabla 3, pero insignificante 
cuando se emplean dichos estimadores. Sobre este punto regresaremos en el siguiente apartado. La segunda anotación es que, al realizar un análisis de descomposición de la varianza del error de pronóstico para el OROSpot, sobre 12 y 24 periodos mensuales, los regresores USDEER y SP500 contribuyen con al menos el 2\% de la varianza del error de pronóstico desde los periodos iniciales (ver Tabla 6). Estas cifras, basadas en las matrices ortogonales de coeficientes de impulso-respuesta, aunadas al hecho de que las variables del modelo ajustado registran el signo esperado, constituyen argumentos sólidos para concluir que el precio de cierre mensual OROSpot puede ser explicado de manera adecuada por su primer rezago, el primer rezago de USDEER y SP500, así como por el término determinista de tendencia. La forma en que estas variables interactúan es, también, de mucha utilidad en esos pronósticos ya que, como se muestra en la Tabla 7, su correlación es mayoritariamente negativa, esto es: con excepción de la pareja SP500-OROSpot que arroja un valor positivo pero marginal (0.02) no significativo, todas las demás combinaciones son significativas y cercanas al -50\%. Estas características del signo y la magnitud de las correlaciones son importantes tenerlas en mente porque, como ya ha sido ampliamente documentado, favorece el rol del oro como balanceador efectivo de portafolios.

Tabla 6

Contribución a la varianza del error de pronóstico de los factores del VAR ajustado

\begin{tabular}{cccc}
\hline \# Periodos(meses) & OROSpot & USDEER & SP500 \\
\hline 12 & $93.81 \%$ & $3.89 \%$ & $2.29 \%$ \\
24 & $83.20 \%$ & $10.92 \%$ & $5.88 \%$ \\
\hline
\end{tabular}

Fuente: elaboración propia.

Tabla 7

Matriz de coeficientes de correlación lineal entre los rendimientos logarítmicos mensuales de las variables seleccionadas

\begin{tabular}{cccc}
\hline & OROSpot & USDEER & SP500 \\
\hline OROSpot & 1 & -0.45 & 0.02 \\
& & $(1.2 \mathrm{E}-14)$ & $(0.78)$ \\
USDEER & -0.45 & 1 & -0.47 \\
& $(1.2 \mathrm{E}-14)$ & & $(3.8 \mathrm{E}-16)$ \\
SP500 & 0.02 & -0.47 & 1 \\
& $(0.78)$ & $(3.8 \mathrm{E}-16)$ & \\
\hline
\end{tabular}

Nota: Los valores p asociados a una prueba $t$ que contrasta la hipótesis nula de correlación lineal cero con la hipótesis alternativa de 2 colas, aparecen entre paréntesis.

Fuente: elaboración propia. 


\section{Filtraciones GARCH}

Una vez identificadas los determinantes del comportamiento marginal de los rendimientos logarítmicos de OROSpot, resulta importante filtrar las series con alguna especificación GARCH que ayude a pronosticar la media y la varianza condicionales sobre distintos periodos. Para tal efecto consideramos que $p_{1 t}=\ln \left(O R O S p o t_{t}\right), p_{2 t}=\ln \left(U S D E E R_{t}\right) \mathrm{y}$ $p_{3 t}=\ln \left(S P 500_{t}\right)$ son los precios de las series utilizadas en el modelo VAR ajustado y que las pruebas de validación de la Tabla 5 permiten establecer las relaciones causales indicadas en el sistema (2):

$$
\left\{\begin{array}{l}
p_{1 t}=\beta_{10} t+\beta_{11} p_{1, t-1}+\beta_{12} p_{2, t-1}+\beta_{13} p_{3, t-1}+u_{1 t} \\
p_{2 t}=\beta_{20} t+\beta_{22} p_{2, t-1}+\beta_{23} p_{3, t-1}+u_{2 t} \\
p_{3 t}=\beta_{33} p_{3, t-1}+u_{3 t}
\end{array}\right.
$$

donde los términos de error $u_{1 t}, u_{2 t}$ y $u_{3 t}$ con media cero presentan autocorrelación nula, heterocedasticidad y no normalidad en su distribución subyacente. Este sistema puede ser reescrito como (3) al tomar las primeras diferencias de las series de rendimientos logarítmicos $r_{i t}=p_{i t}-p_{i, t-1}, i=1,2,3$ :

$$
\left\{\begin{array}{l}
r_{1 t}=\beta_{10}+\beta_{11} r_{1, t-1}+\beta_{12} r_{2, t-1}+\beta_{13} r_{3, t-1}+u_{1 t}^{*} \\
r_{2 t}=\beta_{20}+\beta_{22} r_{2, t-1}+\beta_{23} r_{3, t-1}+u_{2 t}^{*} \\
r_{3 t}=\beta_{33} r_{3, t-1}+u_{3 t}^{*}
\end{array}\right.
$$

donde $u_{i t}^{*}=u_{i t}-u_{i, t-1}, i=1,2,3$ corresponden a los procesos de error de los rendimientos. Ahora bien, al resolver para $r_{2, t-1}$ y $r_{3, t-1}$ en las últimas dos ecuaciones para luego sustituir el resultado en la primera ecuación tenemos que el rendimiento del OROSpot se puede expresar en términos de su primer rezago y los rendimientos contemporáneos de USDEER y SP500, de la siguiente manera:

$$
r_{1 t}=\beta_{10}^{*}+\beta_{11}^{*} r_{1, t-1}+\beta_{12}^{*} r_{2 t}+\beta_{13}^{*} r_{3 t}+v_{1 t}
$$

para parámetros $\beta_{10}^{*}, \beta_{11}^{*}, \beta_{12}^{*}, \beta_{13}^{*}$ y término de error $v_{1 t}$.

Este resultado deja en claro que las ecuaciones del sistema (2) identifican a los rendimientos de USDEER y SP500 como variables explicativas del comportamiento medio de los rendimientos de OROSpot. Pero también hacen evidente que la endogeneidad inducida por las ecuaciones del modelo VAR ajustado reformulado sobre las diferencias $r_{2 t}$ y $r_{3 t}$ puede 
traducirse en una posible dependencia (en media condicional) entre éstas y $v_{1} t$, así como en una mayor correlación serial y heterocedasticidad. Para tratar con estos problemas, el modelo (4) adopta una forma funcional para la media condicional del rendimiento del oro que facilita el filtrado correcto del término de error, del mismo modo que las dos últimas ecuaciones de (3) utilizan el filtrado de los rendimientos de USDEER y SP500 como regresores externos en la dinámica del rendimiento del oro.

Para descartar dependencias no deseadas o, dicho de otro modo, que las variaciones en los rendimientos del oro no anticipen a las series de rendimientos de USDEER y SP500, la Tabla A2 del Apéndice muestra las matrices de autocorrelación cruzada, en la que cada entrada $(i, j)$ corresponde a la correlación muestral entre $r_{i, t}$ y $r_{j, t-l}$, para los distintos números de rezagos $l$. Ahí se observa que, al considerar el valor crítico \pm 0.122 (para una prueba de dos colas al 5\% de significancia bajo la distribución asintótica de la correlación lineal en presencia de ruido blanco) el OROSpot presenta solo dos correlaciones marginales significativas en los rezagos 7 y 8 . Este resultado junto con los niveles de correlación observados en las Tablas A1 permiten confirmar la conclusión establecida en el modelo VAR: que las series de USDEER y SP500 y el primer rezago de OROSpot anticipan a los rendimientos del oro y no viceversa. De manera complementaria, la Tabla A2 incluye el cálculo de una estadística tipo Portmanteau multivariada propuesta por Li y McLeod (1981) para contrastar la hipótesis nula de que todas las autocorrelaciones cruzadas hasta cierto número de rezagos son cero. Para un nivel de significancia del $5 \%$, la prueba concluye que no existe evidencia para rechazar la hipótesis nula, excepto en un par de casos para 3 y 8 rezagos (de hecho, la conclusión es válida en todos los rezagos analizados para niveles de significancia del $1 \%$ o menos).

Con el fin de capturar el comportamiento de la varianza condicional de $u_{i t}^{*}$ y filtrar adecuadamente la media condicional de los rendimientos del oro decidimos utilizar modelos tipo GARCH univariados y no multivariados, aun cuando la prueba ARCH-LM de la Tabla 5 sugiere que la estimación de un modelo multivariado es una opción factible. Aquí optamos por concentrarnos en los hallazgos del modelo VAR calibrado como argumentos para la selección de los regresores externos y dejar la opción de un GARCH multivariado como línea de investigación futura. El análisis de backtesting implementado más adelante muestra que la estrategia elegida resulta altamente efectiva para describir las variaciones futuras del oro.

En el contexto univariado, se dice que el proceso $\left(y_{t}\right)$ sigue un modelo de media y varianza condicionales si se puede expresar como

$$
y_{t}=\mu_{t}+\sigma_{t} z_{t}
$$

donde $\left(z_{t}\right)$ es un proceso de ruido blanco, $\mu_{t}=\mu\left(\theta, x_{t}\right)=E\left(y_{t} \mid x_{t}\right)$ la media condicional y $\sigma_{t}^{2}=\sigma^{2}\left(\theta, x_{t}\right)=E\left(\left(y_{t}-\mu_{t}\right)^{2} \mid x_{t}\right)$ la varianza condicional. Las variables $x_{t}$ denotan el conjunto 
de regresores externos y $y_{t}$ la densidad condicional, que usualmente es escrita en términos de ( $\left.\mu_{t}, \sigma_{t}, \vartheta\right)$ donde $\vartheta=\vartheta\left(\theta, x_{t}\right)$ contiene al resto de los parámetros de la distribución (sesgo y forma, posiblemente). Como caso particular, la versión estándar del denominado modelo $\operatorname{GARCH}(1,1)$ se obtiene considerando $\mu_{t}=\mu, \quad \sigma_{t}^{2}=\omega+\alpha \varepsilon_{t-1}^{2}+\beta \sigma_{t-1}^{2}, \varepsilon_{t}=\sigma_{t} z_{t}$. La notación utilizada es consistente con las dinámicas de los modelos GARCH ajustados en las Tablas 9 y 10 .

Con fines ilustrativos iniciaremos con un modelo $\operatorname{GARCH}(1,1)$ estándar que incorpora a USDEER, SP500 y WTI como regresores en las ecuaciones de la media y la varianza condicionales. El propósito de este ejercicio es doble porque, además de iniciar con una especificación base que permita afinar las ecuaciones más predictivas para la media y la varianza de los regresores, ayuda a comprobar, por otro camino, que la introducción de variables previamente descartadas como el WTI no afecta las conclusiones ya alcanzadas, sobre todo cuando se usan estimadores HAC. En este sentido si WTI no resulta significativa con esta nueva especificación entonces puede decirse que los resultados del VAR anterior son robustos y no dependen de un método particular de ajuste.

En la especificación se incluye un término de primer orden para capturar la naturaleza autorregresiva observada en los rendimientos de OROSpot, bajo el entendido que la distribución condicional de los errores es normal estándar. Los resultados de la Tabla 8 indican que los componentes de la varianza condicional para la serie de OROSpot son influenciados exclusivamente por los elementos estructurales de la especificación GARCH utilizada y que, como es de esperarse, solo USDEER y SP500 resultan significativas como variables explicativas de la media condicional. La eliminación secuencial de los componentes no significativos y un análisis general sobre los residuales estandarizados permiten concluir que WTI no es un regresor externo de la ecuación de la media condicional del OROSpot, tal como se había corroborado con el análisis VAR.

Tabla 8

Estimaciones de los parámetros del modelo AR(1)-GARCH(1,1) para OROSpot sobre los rendimientos logarítmicos mensuales del precio del oro en USD

\begin{tabular}{lcccc}
\hline \multicolumn{1}{c}{ Parámetros } & Estimación & Error Estándar & Estadística t & Valor $\mathrm{p}$ \\
\hline Ecuación para la media: & & & & 0.0204 \\
mu & 0.0062 & 0.0027 & 2.3194 & 0.0187 \\
rezago1 $\Delta \ln ($ OROSpot) & -0.1443 & 0.0613 & -2.3518 & 0.0000 \\
$\Delta \ln (\mathrm{USEER})$ & -1.5699 & 0.2313 & -6.7864 & 0.0006 \\
$\Delta \ln ($ SP500) & -0.2283 & 0.0662 & -3.4465 & 0.1922 \\
$\Delta \ln ($ WTI $)$ & 0.0389 & 0.0298 & 1.3042 &
\end{tabular}


Ecuación para la varianza:

$\begin{array}{lllll}\text { omega } & 0.0006 & 0.0002 & 2.2877 & 0.0222 \\ \text { alpha1 } & 0.2434 & 0.2791 & 0.8719 & 0.3832 \\ \text { beta1 } & 0.4237 & 0.2956 & 1.4332 & 0.1518 \\ \Delta \ln (\text { USEER }) & 0.0000 & 0.0089 & 0.0000 & 1.0000 \\ \Delta \ln (\text { SP500 }) & 0.0000 & 0.0048 & 0.0000 & 1.0000 \\ \Delta \ln (\text { WTI }) & 0.0000 & 0.0020 & 0.0000 & 1.0000\end{array}$

Nota: las estimaciones de las ecuaciones son obtenidas por máxima verosimilitud. Fuente: elaboración propia

El problema consiste ahora en determinar qué criterios son adecuados para elegir la mejor especificación GARCH que modele marginalmente la serie de rendimientos logarítmicos mensuales del OROSpot. El asunto no es menor, sobre todo si consideramos la amplia gama de modelos GARCH utilizados en la literatura. Así que para minimizar las arbitrariedades en la elección, se consideran aquellas especificaciones que satisfagan los siguientes tres criterios:

C1. Los coeficientes de los términos estructurales en la ecuación de la media y la varianza condicionales sean significativos;

C2. Las pruebas estadísticas para la validación de los supuestos de las series de residuales estandarizados y de especificación del modelo sean satisfactorias;

C3. Los análisis de desempeño (backtesting) del modelo seleccionado en el pronóstico de cuantiles de los rendimientos, para distintos niveles de probabilidad y horizontes de riesgo, no sean estadísticamente inferiores a los de los modelos competidores.

Para facilitar la lectura de las Tablas más adelante indicadas y relacionar sus resultados con la ecuación (4), solo basta utilizar la notación $y_{t}=r_{1 t}, x_{1 t}=r_{2 t}$ y $x_{2 t}=r_{3 t} \quad \mathrm{y}$ considerar la inclusión de los parámetros adicionales atribuidos a las diversas especificaciones GARCH. Ahora bien, ya que en este apartado se consideran solamente los criterios C1 y C2, la Tabla 9 presenta las estimaciones para las filtraciones marginales de cada serie de rendimientos logarítmicos mensuales del OROSpot, USDEER y SP500. 
Tabla 9

Resultados de estimación de los parámetros asociados a las diversas especificaciones GARCH calibradas para los rendimientos logarítmicos mensuales $y_{t}$ de OROSpot

\begin{tabular}{|c|c|c|c|c|c|c|c|c|c|c|c|}
\hline \multicolumn{6}{|c|}{$\begin{array}{l}y_{t}=\mu+\psi_{1} y_{t-1}+\lambda \sigma_{t}+\rho_{1} x_{1 t}+\rho_{2} x_{2 t}+\varepsilon_{t} \\
\varepsilon_{t}=\sigma_{t} z_{t}\end{array}$} & \multicolumn{6}{|c|}{$\begin{array}{l}\text { GJRGARCH(1,1): } \sigma_{t}^{2}=\omega+\alpha \varepsilon_{t-1}^{2}+\gamma 1_{\left(\varepsilon_{t-1}<0\right)} \varepsilon_{t-1}^{2}+\beta \sigma_{t-1}^{2} \\
\operatorname{EGARCH}(1,1): \log \left(\sigma_{t}^{2}\right)=\omega+\alpha z_{t-1}+\gamma\left|z_{t-1}\right|+\beta \log \left(\sigma_{t-1}^{2}\right)\end{array}$} \\
\hline Especificación & $\mu$ & $\psi_{1}$ & $\lambda$ & $\rho_{1}$ & $\rho_{2}$ & $\omega$ & $\alpha$ & $\beta$ & $\gamma$ & $\xi$ & v \\
\hline $\operatorname{AR}(\mathbf{1})-\operatorname{GARCH}(\mathbf{1}, \mathbf{1})$ & $\begin{array}{l}6.03 E-03 \\
(2.17 E-03)\end{array}$ & $\begin{array}{c}-0.15 \\
(5.46 \mathrm{E}-02)\end{array}$ & & $\begin{array}{c}-1.64 \\
(2.30 \mathrm{E}-01)\end{array}$ & $\begin{array}{c}-0.22 \\
(7.18 E-02)\end{array}$ & $\begin{array}{l}4.79 \mathrm{E}-04 \\
(2.54 \mathrm{E}-04)\end{array}$ & $\begin{array}{c}0.19 \\
(1.11 \mathrm{E}-01)\end{array}$ & $\begin{array}{c}0.52 \\
(1.87 \mathrm{E}-01)\end{array}$ & & 1 & $\begin{array}{c}11.43 \\
(6.28 E+00)\end{array}$ \\
\hline AR(1)-EGARCH(1,1) & $\begin{array}{l}5.85 \mathrm{E}-03 \\
(9.23 \mathrm{E}-04)\end{array}$ & $\begin{array}{c}-0.16 \\
(4.49 E-02)\end{array}$ & & $\begin{array}{l}-1.64 \\
(1.49 \mathrm{E}-01)\end{array}$ & $\begin{array}{c}-0.23 \\
(2.46 \mathrm{E}-02)\end{array}$ & $\begin{array}{c}-2.06 \\
(1.40 \mathrm{E}+00)\end{array}$ & $\begin{array}{c}0.04 \\
(1.20 \mathrm{E}-01)\end{array}$ & $\begin{array}{c}0.68 \\
(2.15 E-01)\end{array}$ & $\begin{array}{c}0.38 \\
(1.55 \mathrm{E}-01)\end{array}$ & 1 & $\begin{array}{l}12.93 \\
(8.28 \mathrm{E}+00)\end{array}$ \\
\hline AR(1)-GJRGARCH(1,1) & $\begin{array}{l}5.99 \mathrm{E}-03 \\
(2.44 \mathrm{E}-03)\end{array}$ & $\begin{array}{c}-0.15 \\
(6.10 \mathrm{E}-02)\end{array}$ & & $\begin{array}{c}-1.64 \\
(2.84 \mathrm{E}-01)\end{array}$ & $\begin{array}{l}-0.22 \\
(7.45 \mathrm{E}-02)\end{array}$ & $\begin{array}{l}4.60 \mathrm{E}-04 \\
(6.04 \mathrm{E}-04)\end{array}$ & $\begin{array}{l}0.18 \\
(3.93 E-01)\end{array}$ & $\begin{array}{c}0.54 \\
(5.68 \mathrm{E}-01)\end{array}$ & $\begin{array}{l}0.02 \\
(3.73 \mathrm{E}-01)\end{array}$ & 1 & $\begin{array}{c}11.29 \\
(8.30 \mathrm{E}+00)\end{array}$ \\
\hline$\stackrel{\vec{\partial}}{A R(1)-\operatorname{IGARCH}(1,1)}$ & $\begin{array}{l}6.72 \mathrm{E}-03 \\
(2.78 \mathrm{E}-03)\end{array}$ & $\begin{array}{l}-0.15 \\
(7.18 E-02)\end{array}$ & & $\begin{array}{l}-1.61 \\
(2.45 E-01)\end{array}$ & $\begin{array}{l}-0.24 \\
(9.80 \mathrm{E}-02)\end{array}$ & & $\begin{array}{c}0.03 \\
(3.29 \mathrm{E}-02)\end{array}$ & 0.97 & & 1 & $\begin{array}{c}10.56 \\
(5.36 \mathrm{E}+00)\end{array}$ \\
\hline $\operatorname{AR}(1)-\operatorname{GARCH}(1,1)$ in-mean & & $\begin{array}{l}-0.15 \\
(5.55 E-02) \\
\end{array}$ & $\begin{array}{c}0.16 \\
(5.32 \mathrm{E}-02) \\
\end{array}$ & $\begin{array}{l}-1.63 \\
(2.29 \mathrm{E}-01) \\
\end{array}$ & $\begin{array}{c}-0.23 \\
(7.4 \mathrm{E}-02) \\
\end{array}$ & $\begin{array}{l}4.86 \mathrm{E}-04 \\
(2.75 \mathrm{E}-04)\end{array}$ & $\begin{array}{c}0.19 \\
(1.10 \mathrm{E}-01)\end{array}$ & $\begin{array}{c}0.52 \\
(1.95 E-01)\end{array}$ & & 1 & $\begin{array}{c}11.21 \\
(5.98 E+00) \\
\end{array}$ \\
\hline 㩊 $\overline{\left(x_{1 t}\right): \text { AR(1)-GARCH(1,1) }}$ & & $\begin{array}{c}0.11 \\
(6.20 \mathrm{E}-02)\end{array}$ & & & & $\begin{array}{l}2.66 \mathrm{E}-05 \\
(1.62 \mathrm{E}-05)\end{array}$ & $\begin{array}{c}0.12 \\
(5.76 \mathrm{E}-02)\end{array}$ & $\begin{array}{c}0.76 \\
(1.14 E-01)\end{array}$ & & $\begin{array}{c}1.15 \\
(8.47 \mathrm{E}-02)\end{array}$ & $\begin{array}{c}9.27 \\
(4.44 \mathrm{E}+00)\end{array}$ \\
\hline 道 $\left(x_{2 t}\right): \operatorname{GARCH}(1,1)$ & $\begin{array}{l}8.34 \mathrm{E}-03 \\
(1.94 \mathrm{E}-03)\end{array}$ & & & & & $\begin{array}{l}7.33 \mathrm{E}-05 \\
(4.70 \mathrm{E}-05)\end{array}$ & $\begin{array}{c}0.22 \\
(4.73 E-02)\end{array}$ & $\begin{array}{c}0.75 \\
(5.53 E-02)\end{array}$ & & $\begin{array}{c}0.61 \\
(6.93 \mathrm{E}-02)\end{array}$ & $\infty$ \\
\hline
\end{tabular}

Nota: La media condicional de $y_{t}$ incorpora los rendimientos logarítmicos mensuales de USDEER $\left(x_{1 t}\right)$ y SP500 $\left(x_{2 t}\right)$. Las estimaciones son obtenidas mediante el método de máxima verosimilitud. Los valores entre paréntesis son los errores estándar robustos basados en el método de White (1982). En el encabezado se han incluido expresiones generales abreviadas sobre las dinámicas asociadas a los procesos de rendimientos y su volatilidad condicional $\sigma_{t}$ bajo cada modelo. Se incluyen también los resultados de la estimación del filtrado individual de las series de regresores externos ( $x_{1 t}$ y $x_{2 t}$ ). En todos los casos, la distribución condicional utilizada es te Student con parámetros de forma $v$ y sesgo $\xi$ (el valor $v=\infty$ corresponde la distribución normal estándar sesgada). La distribución es simétrica cuando $\xi<1$.

Fuente: elaboración propia.

Ahí se observa, en general, que: el componente de varianza condicional para las series de rendimientos de OROSpot exhibe menor persistencia que el de los regresores externos USDEER y SP500; el proceso de ruido blanco $\left(z_{\boldsymbol{t}}\right)$ asociado al OROSpot es simétrico y presenta cierto grado de pesadez en ambas colas, tal como se desprende de los parámetros de forma $(v)$ y sesgo $(\xi)$ de la distribución condicional; el factor USDEER exhibe cierto grado de asimetría positiva con tendencia a tomar valores extremos; $\mathrm{y}$, finalmente, el proceso de ruido asociados a SP500 es normal estándar $(v=\infty)$, con sesgo negativo $(\xi<1)$. Con respecto a la estructura de la media condicional de las series de rendimientos logarítmicos, la Tabla 9 muestra un efecto autorregresivo de orden uno para el OroSpot (cuyo efecto parcial es de -0.15), así como contribuciones negativas importantes provenientes de los términos contemporáneos de los regresores externos USDEER (-1.63) y SP500 (-0.23). Al incorporarse el efecto contemporáneo de la volatilidad condicional en la media del proceso de rendimientos se observa un cambio de signo en el efecto parcial asociado (0.16), contrario al efecto autorregresivo, que permite filtrar el impacto de los periodos de elevada volatilidad como incrementos en el rendimiento esperado del OROSpot. Los regresores externos exhiben comportamiento autorregresivo con signo positivo (+0.11), en el caso de USDEER, y media constante positiva (+0.0083), en el caso de SP500. 
En cuanto a la calibración de las estructuras para la varianza condicional cabe destacar que, bajo la especificación GARCH estándar, el efecto autorregresivo que induce el rezago de orden 1 del componente estructural de la varianza condicional para el OROSpot (0.52) es menor a los registrados por USDEER (0.76) y SP500 (0.75). Este efecto es superior a la contribución diferenciada que tiene el primer rezago cuadrado del proceso de error sobre la dinámica de la varianza condicional de las tres series ( 0.19 en OROSpot, 0.12 para USDEER y 0.22 para SP500). También es de notar que si bien el coeficiente que captura el denominado efecto apalancamiento resulta positivo para la especificación EGARCH, no lo es para GJRGARCH, porque ahí el coeficiente es significativamente cercano a cero. Finalmente, todos los modelos ajustados, con excepción del IGARCH, resultan estacionarios, aun cuando la persistencia promedio que se observa para el proceso de volatilidad condicional de OROSpot (0.71) se encuentra muy por debajo de los patrones más explosivos identificados con los regresores externos USDEER (0.87) y SP500 (0.97).

Para validar los supuestos de las series de residuales, de los elementos estructurales y de las especificaciones asociadas a cada modelo ajustado, se presentan varias pruebas estadísticas en la Tabla 10. Ahí se puede observar que, además de la persistente volatilidad identificada para cada modelo, también se puede agregar que las especificaciones calibradas del OROSpot no presentan mayores problemas de estabilidad en los parámetros, debido a que los valores críticos (al 10\% de significancia) asociados a las estadísticas de prueba (Nyb) son del orden de 1.89 (GARCH y GARCH-in-mean), 2.10 (EGARCH, GJRGARCH) y 1.49 (EWMA). Esta situación es distinta para los regresores externos USDEER y SP500, ya que sus correspondientes valores críticos son 1.49 y 1.28 , respectivamente. Los niveles de sesgo y curtosis que presentan los residuales estandarizados bajo cada especificación se encuentran en correspondencia con las estimaciones reportadas para los parámetros de asimetría ( $\xi$ ) y forma $(v)$ de la distribución condicional seleccionada. Por otro lado, los valores p de la prueba HL confirman la correcta especificación de la distribución condicional del proceso de ruido blanco $\left(z_{t}\right)$ en todos los casos, con excepción de la especificación EWMA. 
Tabla 10

Pruebas estadísticas para la validación de supuestos de las especificaciones GARCH

\begin{tabular}{|c|c|c|c|c|c|c|c|c|c|c|c|c|c|c|c|c|}
\hline \multicolumn{4}{|c|}{$\begin{array}{l}y_{t}=\mu+\psi_{1} y_{t-1}+\lambda \sigma_{t}+\rho_{1} x_{1 t}+\rho_{2} x_{2 t}+\varepsilon_{t} \\
\varepsilon_{t}=\sigma_{t} z_{t} \\
z_{t} \sim t_{v, \xi}(0,1) \text { i.i.d. }\end{array}$} & \multicolumn{6}{|c|}{$\operatorname{GARCH}(1,1): \sigma_{t}^{2}=\omega+\alpha \varepsilon_{t-1}^{2}+\beta \sigma_{t-1}^{2}$} & \multicolumn{7}{|c|}{$\begin{array}{l}\operatorname{GJRGARCH}(1,1): \sigma_{t}^{2}=\omega+\alpha \varepsilon_{t-1}^{2}+\gamma 1_{\left(\varepsilon_{t-1}<0\right)} \varepsilon_{t-1}^{2}+\beta \sigma_{t-1}^{2} \\
\operatorname{EGARCH}(1,1): \log \left(\sigma_{t}^{2}\right)=\omega+\alpha z_{t-1}+\gamma\left|z_{t-1}\right|+\beta \log \left(\sigma_{t-1}^{2}\right)\end{array}$} \\
\hline Especificación & Pers & Nyb & sk & ku & HL & LjB1 & LjB2 & LjBAbs & Sb & Nsb & Psb & Jsb & $\mathrm{Pc}$ & $\mathrm{Pi}$ & BIC & Lik \\
\hline$\overline{\text { AR(1)-GARCH(1.1) }}$ & 0.71 & 2.01 & 0.30 & 3.58 & 0.26 & 0.89 & 0.63 & 0.86 & 0.02 & 0.69 & 0.26 & 0.05 & 0.87 & 0.21 & -3.49 & $4.976 \mathrm{E}+02$ \\
\hline AR(1)-EGARCH(1,1) & 0.68 & 2.06 & 0.28 & 3.48 & 0.14 & 0.78 & 0.59 & 0.74 & 0.02 & 0.59 & 0.19 & 0.10 & 0.87 & 0.21 & -3.48 & 4.984E+02 \\
\hline 蒿 AR(1)-GJRGARCH(1,1) & 0.72 & 2.12 & 0.31 & 3.58 & 0.27 & 0.88 & 0.66 & 0.91 & 0.02 & 0.69 & 0.28 & 0.05 & 0.87 & 0.21 & -3.47 & $4.977 E+02$ \\
\hline ठैّ AR(1)-IGARCH(1,1) & 1.00 & 1.34 & 0.28 & 3.85 & 0.03 & 0.98 & 0.24 & 0.06 & 0.03 & 0.08 & 0.88 & 0.08 & 0.65 & 0.74 & -3.51 & $4.938 E+02$ \\
\hline AR(1)-GARCH(1,1) in-mean & 0.71 & 2.05 & 0.30 & 3.59 & 0.22 & 0.91 & 0.66 & 0.91 & 0.04 & 0.77 & 0.39 & 0.10 & 0.87 & 0.21 & -3.49 & $4.975 \mathrm{E}+02$ \\
\hline 总 $\left(x_{1 t}\right):$ AR(1)-GARCH(1,1) & 0.87 & 1.03 & 0.47 & 3.85 & 0.91 & 0.57 & 0.52 & 0.43 & 0.93 & 0.41 & 0.96 & 0.78 & 0.65 & 0.27 & -5.56 & $7.734 E+02$ \\
\hline 总 $\left(x_{2 t}\right): \operatorname{GARCH}(1,1)$ & 0.97 & 0.76 & -0.88 & 4.51 & 0.21 & 0.76 & 0.61 & 0.31 & 0.66 & 0.69 & 0.07 & 0.07 & 0.87 & 0.62 & -3.65 & $5.106 E+02$ \\
\hline
\end{tabular}

Nota: Para cada modelo ajustado se reportan: la persistencia (Pers) de la volatilidad; la estadística de la prueba de estabilidad de parámetros (Nyb) de Nyblom (1989); el sesgo (sk) y curtosis (ku) de los residuales estandarizados; el valor-p de la prueba de densidad no paramétrica (HL) de Hong y Li (2005) para evaluar el grado de ajuste de la distribución condicional; los valores-p asociados a las estadísticas de Ljung-Box para detectar si existe presencia de autocorrelación serial en los residuales estandarizados ( $\mathrm{LjB} 1)$, así como para sus valores al cuadrado (LjB2) y valores absolutos (LjBAbs); los valores-p de las pruebas de sesgo de signo de Engle y Ng (1993) para identificar el tipo de efecto en los residuales estandarizados del modelo ante cambios (shocks) en la volatilidad en los casos de sesgo positivo ( $\mathrm{Sb}$ ), sesgo negativo (Nb) y la prueba conjunta asociada (Jsb); los valores-p de las pruebas de cobertura incondicional (Pc) e independencia (Pi) propuestas por Christoffersen (1998) considerando un intervalo de confianza del $99 \%$ sobre la cola izquierda de los rendimientos. Finalmente, se incluyen los valores del criterio de información Bayesiano (BIC) y la verosimilitud no normalizada (Lik).

Fuente: elaboración propia

El cálculo de los valores p asociados a las estadísticas de Ljung-Box, y que son usados para validar la hipótesis de no autocorrelación serial de los residuales estandarizados, ya sean nominales ( $\mathrm{LjB} 1)$ cuadrados ( $\mathrm{LjB} 2$ ) o en valor absoluto ( $\mathrm{LjBAbs}$ ), indica que el filtrado de los rendimientos es adecuado. Del mismo modo, la mayoría de las pruebas de sesgo de signo apoyan la idea de que no existe evidencia de que las especificaciones tengan problemas de sesgo para capturar la volatilidad, pues con excepción de la serie para el OROSpot que exhibe sesgo de signo ( $\mathrm{Sb}$ ), no hay otros problemas de asimetría ante cambios abruptos o shocks. Los resultados de la prueba de cobertura condicional $(\mathrm{Pc})$ implementada para el cálculo del Valor en Riesgo (VaR) al 99\% sobre la cola izquierda de los rendimientos (dentro de la muestra), sostienen que el número de excepciones observadas (pérdidas superiores al VaR) se encuentra en línea con el nivel de confianza de la métrica de riesgo en todos los casos.

Con todos estos resultados y considerando los criterios de información BIC y el de verosimilitud (Lik), los modelos GARCH estándar y EGARCH son, respectivamente, los que tienen mayor capacidad predictiva para modelar la serie OROSpot.

\section{Pronósticos sobre múltiples periodos futuros}

Con el propósito de evaluar el desempeño de las especificaciones de filtrado tipo GARCH en el pronóstico del OROSpot de manera marginal, como se indica en el criterio C3, se im- 
plementa a continuación un mecanismo de backtesting global. Es decir, se busca reproducir las condiciones representadas por los modelos de la Tabla 9 para pronosticar el rendimiento mensual de las variables de interés sobre varios periodos futuros. Específicamente, el análisis se centra en evaluar el pronóstico de cuantiles en ambas colas de la distribución marginal subyacente para lo cual se fijan los niveles de probabilidad de 1\%, 5\%, 10\%, 15\%, 85\%, $90 \%, 95 \%$ y $99 \%$. En el caso de los cuantiles ubicados en la cola izquierda de la distribución de rendimientos (niveles de 1\%,5\%,10\%,15\%) se compara el número de realizaciones que resultan menores al pronóstico (excesos) con el número esperado de excesos bajo el nivel de probabilidad del cuantil. Para los cuantiles de la cola derecha (niveles de 85\%, 90\%, 95\%, $99 \%$ ) se contrasta el número de rendimientos realizados que se encuentran por encima del pronóstico del cuantil con el número esperado de excesos bajo el nivel correspondiente. Ambos análisis se realizan para cada cuantil y horizonte de periodos hacia adelante.

El número de períodos mensuales $(\mathrm{M})$ hacia adelante que se contemplan son: $1 \mathrm{M}, 2 \mathrm{M}$, $3 \mathrm{M}, 6 \mathrm{M}, 9 \mathrm{M}$ y 12M. Como criterio de reajuste del modelo se establece que cada 6 meses se recalculan los valores de los parámetros de acuerdo con la información histórica disponible hasta ese momento. El proceso de evaluación considera que, para cada horizonte y nivel de probabilidad, se generan pronósticos de la métrica de riesgo VaR durante los últimos 100 periodos mensuales disponibles (conjunto de prueba) en la muestra. Cada pronóstico se debe contrastar con el rendimiento mensual observado (realizado) en el periodo (futuro) pronosticado.

Para medir el grado de correspondencia entre los pronósticos de los cuantiles y los valores realizados de los rendimientos utilizamos la prueba de cobertura no condicional de Kupiec (1995). La Tabla 11 reporta los valores p correspondientes a las estadísticas de esa prueba que, como se sabe, siguen una distribución ji-cuadrada con un grado de libertad bajo la hipótesis nula de que la proporción de excesos observados es igual al nivel de confianza asociado al cuantil (cobertura correcta). En color rojo se etiquetan los casos en que el valor p es menor al nivel de 0.05 de significancia. A la derecha de cada valor p se incluye el número de excesos observados en el área de la cola de la distribución de rendimientos subyacente. De manera complementaria, en el extremo derecho del encabezado de cada tabla se muestra el cálculo de una métrica de distancia que mide el nivel de dispersión promedio que presentan el número de excesos observados con respecto del número de excesos esperados. Este cómputo se hace para cada especificación GARCH.

La conclusion general de la Tabla es que, si consideramos el número de casos en el que se rechaza la hipótesis nula de que el cálculo del cuantil presenta una cobertura correcta, se observa que las especificaciones con mejor desempeño en el pronóstico de cuantiles para la serie OROSpot son AR(1)-GARCH(1,1) y AR(1)-IGARCH(1,1). 
Tabla 11

Resultados de la implementación de la prueba de Kupiec (1995) sobre los pronósticos de cuantiles de rendimientos logarítmicos mensuales de OROSpot bajo diferentes niveles de probabilidad y horizontes de riesgo (periodos hacia adelante).

\begin{tabular}{|c|c|c|c|c|c|c|c|c|c|c|c|c|c|c|c|c|c|c|c|c|c|c|c|c|}
\hline & \multicolumn{12}{|c|}{ AR(1)-GARCH(1,1)-t Student } & \multicolumn{12}{|c|}{ AR(1)-EGARCH(1,1)-t Student } \\
\hline \begin{tabular}{|l|} 
Nivel \\
\end{tabular} & \multicolumn{2}{|c|}{$1 \mathrm{M}$} & \multicolumn{2}{|c|}{$\mathbf{2 M}$} & \multicolumn{2}{|c|}{$3 M$} & \multicolumn{2}{|c|}{$6 M$} & \multicolumn{2}{|c|}{$9 M$} & \multicolumn{2}{|c|}{$12 M$} & \multicolumn{2}{|c|}{$1 \mathrm{M}$} & \multicolumn{2}{|c|}{$2 M$} & \multicolumn{2}{|c|}{$3 \mathrm{M}$} & \multicolumn{2}{|c|}{$6 M$} & \multicolumn{2}{|c|}{$9 M$} & \multicolumn{2}{|l|}{$12 \mathrm{M}$} \\
\hline $1 \%$ & 0.10 & & 0.38 & & 0.10 & 3 & 0.10 & 3 & 0.10 & 3 & 0.10 & 3 & 0.10 & 3 & 0.02 & 41 & 0.10 & 3 & 0.10 & 3 & 0.10 & 3 & 0.10 & 3 \\
\hline $5 \%$ & 0.02 & 11 & 0.04 & 10 & 0.02 & 11 & 0.10 & 9 & 0.10 & 9 & 0.01 & 12 & 0.01 & 12 & 0.04 & 10 & 0.01 & 12 & 0.01 & 12 & 0.00 & 13 & 0.00 & 14 \\
\hline $10 \%$ & 0.21 & 14 & 0.06 & 16 & 0.12 & 15 & 0.02 & 18 & 0.01 & 19 & 0.03 & 17 & 0.12 & 15 & 0.06 & 16 & 0.06 & 16 & 0.02 & 18 & 0.01 & 19 & 0.01 & 19 \\
\hline $15 \%$ & 0.18 & 20 & 0.41 & 18 & 0.28 & 19 & 0.41 & 18 & 0.18 & 20 & 0.11 & 21 & 0.28 & 19 & 0.18 & 20 & 0.11 & 21 & 0.18 & 20 & 0.11 & 21 & 0.06 & 22 \\
\hline $85 \%$ & 0.11 & 21 & 0.58 & 17 & 0.18 & 20 & 0.11 & 21 & 0.18 & 20 & 0.06 & 22 & 0.18 & 20 & 0.11 & 21 & 0.02 & 24 & 0.06 & 22 & 0.06 & 22 & 0.04 & 23 \\
\hline $90 \%$ & 0.21 & 14 & 0.34 & 13 & 0.74 & 11 & 0.21 & 14 & 0.12 & 15 & 0.12 & 15 & 0.03 & 17 & 0.03 & 17 & 0.21 & 14 & 0.12 & 15 & 0.06 & 16 & 0.03 & 17 \\
\hline $95 \%$ & 0.39 & 7 & 0.10 & 9 & 0.20 & 8 & 0.20 & 8 & 0.39 & 7 & 0.20 & & 0.39 & 7 & 0.10 & 9 & 0.10 & 9 & 0.10 & 9 & 0.10 & 9 & 0.10 & 9 \\
\hline 99\% & 1.00 & 1 & 0.38 & 2 & 0.38 & 2 & 0.38 & 2 & 1.00 & 1 & 1.00 & 1 & 0.38 & 2 & 0.10 & 3 & 0.38 & 2 & 0.10 & 3 & 0.02 & 4 & 0.02 & 4 \\
\hline
\end{tabular}

\begin{tabular}{|c|c|c|c|c|c|c|c|c|c|c|c|c|c|c|c|c|c|c|c|c|c|c|c|c|}
\hline & \multicolumn{12}{|c|}{ AR(1)-GJRGARCH(1,1)t Student } & \multicolumn{12}{|c|}{ AR(1)-IGARCH(1,1)-t Student } \\
\hline Nivel & \multicolumn{2}{|c|}{$\mathbf{1 M}$} & \multicolumn{2}{|c|}{$\mathbf{2 M}$} & \multicolumn{2}{|c|}{$3 M$} & \multicolumn{2}{|c|}{$6 \mathrm{M}$} & \multicolumn{2}{|c|}{$9 M$} & \multicolumn{2}{|c|}{$12 \mathrm{M}$} & \multicolumn{2}{|c|}{$1 \mathrm{M}$} & \multicolumn{2}{|c|}{$2 \mathrm{M}$} & \multicolumn{2}{|c|}{$3 \mathrm{M}$} & \multicolumn{2}{|c|}{$6 \mathrm{M}$} & \multicolumn{2}{|c|}{ 9M } & \multicolumn{2}{|c|}{$12 \mathrm{M}$} \\
\hline $1 \%$ & 0.10 & & 0.10 & 3 & 0.10 & 3 & 0.10 & 3 & 0.10 & 3 & 0.10 & 3 & 1.00 & 1 & 0.38 & 2 & 0.38 & 2 & 0.10 & 3 & 0.10 & 3 & 0.10 & 3 \\
\hline $5 \%$ & 0.02 & 11 & 0.01 & 12 & 0.02 & 11 & 0.20 & 8 & 0.04 & 10 & 0.02 & 11 & 0.20 & 8 & 0.39 & 7 & 0.66 & 6 & 0.66 & 6 & 0.66 & 6 & 0.66 & 6 \\
\hline $10 \%$ & 0.21 & 14 & 0.06 & 16 & 0.06 & 16 & 0.02 & 18 & 0.01 & 19 & 0.01 & 19 & 0.21 & 14 & 0.12 & 15 & 0.12 & 15 & 0.03 & 17 & 0.06 & 16 & 0.06 & 16 \\
\hline $15 \%$ & 0.18 & 20 & 0.18 & 20 & 0.41 & 18 & 0.28 & 19 & 0.18 & 20 & 0.11 & 21 & 0.58 & 17 & 0.41 & 18 & 0.41 & 18 & 0.28 & 19 & 0.18 & 20 & 0.18 & 20 \\
\hline $85 \%$ & 0.11 & 21 & 0.11 & 21 & 0.04 & 23 & 0.11 & 21 & 0.11 & 21 & 0.11 & 21 & 0.78 & 16 & 0.41 & 18 & 0.41 & 18 & 0.58 & 17 & 0.58 & 17 & 0.78 & 16 \\
\hline $90 \%$ & 0.06 & 16 & 0.34 & 13 & 0.74 & 11 & 0.21 & 14 & 0.34 & 13 & 0.21 & 14 & 0.74 & 11 & 1.00 & 10 & 1.00 & 10 & 1.00 & 10 & 0.52 & 12 & 0.74 & 11 \\
\hline $95 \%$ & 0.39 & 7 & 0.10 & 9 & 0.20 & 8 & 0.20 & 8 & 0.10 & 9 & 0.20 & 8 & 1.00 & 5 & 0.64 & 4 & 0.64 & 4 & 0.39 & 7 & 1.00 & 5 & 1.00 & 5 \\
\hline $99 \%$ & 0.38 & 2 & 0.38 & 2 & 0.38 & 2 & 1.00 & 1 & 1.00 & 1 & 1.00 & 1 & 1.00 & 1 & 1.00 & 1 & 1.00 & 1 & 0.16 & 0 & 1.00 & 1 & 1.00 & 1 \\
\hline
\end{tabular}

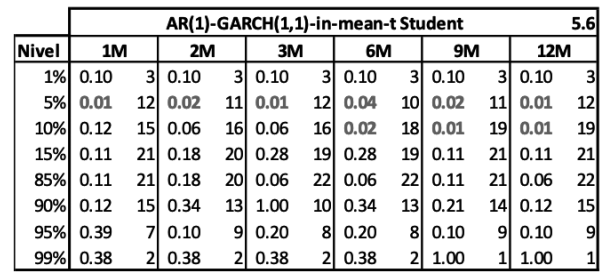

Nota: Para cada horizonte se reporta el valor p de la estadística de prueba (primera columna) y el número de excesos al cuantil (segunda columna) observados en ambas colas. En la esquina superior derecha de cada tabla se reporta el valor promedio sobre los horizontes de riesgo de una métrica de distancia euclidiana que mide el tamaño medio de la dispersión observada entre los excesos realizados y el número de excesos esperados de los cuantiles (en color azul se han etiquetado los dos valores más pequeños). Finalmente, se marcan con color rojo los casos en que el valor p de la estadística de Kupiec es menor al nivel de significancia del 5\%.

Fuente: elaboración propia.

Como estrategia adicional para medir el desempeño de las especifiaciones GARCH empleadas para filtrar los rendimientos de OROSpot se realiza el cálculo de métricas estándar de error de pronóstico de la varianza condicional sobre el conjunto de prueba empleado en el ejercicio de backtesting. Para ello utilizamos las siguientes versiones muestales de funciones de pérdida (criterios) desarrolladas por Hansen y Lunde (2005):

$$
\begin{aligned}
& M S E_{1}=n^{-1} \sum_{i=1}^{n}\left(\sigma_{t}-h_{t}\right)^{2} \\
& M S E_{2}=n^{-1} \sum_{i=1}^{n}\left(\sigma_{t}^{2}-h_{t}^{2}\right)^{2}
\end{aligned}
$$


donde $\left(h_{t}\right)$ es el pronóstico del proceso de volatilidad condicional $\left(\sigma_{t}\right)$ subyacente. Como proxy de la varianza $\sigma_{t}^{2}$, se utiliza el cuadrado del rendimiento mensual realizado.

Tabla 12

Métricas de error de pronóstico $M S E_{1}$ y $M S E_{2}$ bajo cada especificación GARCH sobre diferentes horizontes de riesgo.

\begin{tabular}{|c|c|c|c|c|c|c|}
\multicolumn{10}{c|}{$\boldsymbol{M S}_{\mathbf{1}}\left(\times 10^{-4}\right)$} \\
\cline { 2 - 7 } AR(1)-GARCH(1,1) & $\mathbf{1 M}$ & $\mathbf{2 M}$ & $\mathbf{3 M}$ & $\mathbf{6 M}$ & $\mathbf{9 M}$ & $\mathbf{1 2 M}$ \\
\cline { 2 - 7 } & 8.90 & 9.93 & 10.01 & 9.71 & 9.83 & 9.64 \\
AR(1)-EGARCH(1,1) & $(1.00)$ & $(0.38)$ & $(0.06)$ & $(0.19)$ & $(0.06)$ & $(0.26)$ \\
& 9.02 & 9.65 & 9.59 & 9.32 & 9.33 & 9.33 \\
AR(1)-GJRGARCH(1,1) & $(1.00)$ & $(1.00)$ & $(1.00)$ & $(1.00)$ & $(1.00)$ & $(1.00)$ \\
& 8.93 & 9.71 & 9.73 & 9.39 & 9.35 & 9.17 \\
AR(1)-IGARCH(1,1) & $(1.00)$ & $(1.00)$ & $(1.00)$ & $(1.00)$ & $(1.00)$ & $(1.00)$ \\
& 9.44 & 9.63 & 9.68 & 9.68 & 9.68 & 9.64 \\
AR(1)-GARCH(1,1)-in-mean & $(0.18)$ & $(1.00)$ & $(1.00)$ & $(0.90)$ & $(0.92)$ & $(0.75)$ \\
& 8.89 & 9.70 & 9.75 & 9.50 & 9.40 & 9.23 \\
& $(1.00)$ & $(1.00)$ & $(1.00)$ & $(1.00)$ & $(1.00)$ & $(1.00)$ \\
\hline
\end{tabular}

\begin{tabular}{r|c|c|c|c|c|c|}
\multicolumn{10}{c}{} & \multicolumn{1}{c}{$\boldsymbol{M S}_{\mathbf{2}}\left(\times 10^{-5}\right)$} \\
\cline { 2 - 7 } AR(1)-GARCH(1,1) & $\mathbf{1 M}$ & $\mathbf{2 M}$ & $\mathbf{3 M}$ & $\mathbf{6 M}$ & $\mathbf{9 M}$ & $\mathbf{1 2 M}$ \\
\cline { 2 - 7 } & 1.13 & 1.24 & 1.26 & 1.25 & 1.25 & 1.23 \\
AR(1)-EGARCH(1,1) & $(1.00)$ & $(0.55)$ & $(0.05)$ & $(0.07)$ & $(0.13)$ & $(0.32)$ \\
& 1.17 & 1.25 & 1.26 & 1.26 & 1.26 & 1.27 \\
AR(1)-GJRGARCH(1,1) & $(0.35)$ & $(0.26)$ & $(0.24)$ & $(0.19)$ & $(0.13)$ & $(0.08)$ \\
& 1.16 & 1.24 & 1.24 & 1.22 & 1.22 & 1.20 \\
AR(1)-IGARCH(1,1) & $(0.82)$ & $(0.47)$ & $(0.65)$ & $(1.00)$ & $(1.00)$ & $(1.00)$ \\
AR(1)-GARCH(1,1)-in-mean & 1.15 & 1.17 & 1.17 & 1.17 & 1.17 & 1.17 \\
& $(1.00)$ & $(1.00)$ & $(1.00)$ & $(1.00)$ & $(1.00)$ & $(1.00)$ \\
& 1.15 & 1.24 & 1.25 & 1.24 & 1.24 & 1.22 \\
$(1.00)$ & $(0.63)$ & $(0.43)$ & $(0.52)$ & $(0.60)$ & $(0.96)$ \\
\hline
\end{tabular}

Nota: Los valores entre paréntesis denotan el valor $\mathrm{p}$ de la prueba de capacidad predictiva superior (SPA) de Hansen (2005).

Fuente: elaboración propia.

El cálculo de los criterios $M S E_{1}$ y $M S E_{2}$ permite evaluar el error de pronóstico en la volatilidad para cada uno de los horizontes mensuales $1 \mathrm{M}, 2 \mathrm{M}, 3 \mathrm{M}, 6 \mathrm{M}, 9 \mathrm{M}$ y $12 \mathrm{M}$ bajo cada modelo tipo GARCH utilizado en el ejercicio de backtesting. Para verificar si los pronósticos son estadísticamente diferentes se utiliza la prueba de capacidad predictiva superior (SPA) propuesta por Hansen (2005) en la que la hipótesis nula establece que ningun modelo es inferior a otros modelos competidores dada una función de pérdida. En la Tabla 12 se muestran los valores obtenidos para los criterios $M S E_{1}$ y $M S E_{2}$ en cada especificación GARCH bajo cada horizonte de pronóstico considerado. Entre p aréntesis se incluyen los valores $\mathrm{p}$ que resultan de la aplicación de la prueba SPA. En color rojo se han identificado los casos en que el valor $\mathrm{p}$ es inferior al nivel 0.05 de significancia. En general, no existen diferencias 
significativas en la capacidad predictiva de la volatilidad, excepto para las especificaciones $\operatorname{AR}(1)-G A R C H(1,1)$ y $\operatorname{IGARCH}(1,1)$ con horizontes de 3 y 6 meses, respectivamente. Si se comparan los valores promedio de cada métrica utilizada se observa que bajo el criterio $M S E_{1}$ los modelos tipo EGARCH presentan los menores niveles de errores. En el mismo sentido, pero con base en el criterio $M S E_{2}$, la especificación tipo IGARCH mantienen los promedios de error más pequeños.

Una vez analizados los resultados de validación (Tablas 9 a 12) sobre los aspectos considerados en los criterios C1, C2 y C3 descritos anteriormente, se concluye que la especificación más adecuada para el filtrado de los rendimientos logarítmicos mensuales de OROSpot resulta ser el modelo AR(1)-GARCH(1,1) con distribución t de Student y regresores externos USDEER y SP500.

\section{Conclusion}

El documento ofrece suficiente evidencia para afirmar que las fluctuaciones del precio del oro experimentadas entre Enero de 1995 y Agosto 2017 pueden ser explicadas, básicamente, por su primer rezago y el de los regresores externos USDEER y SP500. El analisis VAR y las diversas pruebas ensayadas a lo largo de las Tablas 9 a 12 garantizan una bien fundamentada causalidad entre los dos factores y los rendimientos del OROSpot (y en una sola direccion), que permite asegurar que las inversiones en oro son, durante ese periodo, un medio eficiente de cobertura contra las volatilidades cambiarias (USDEER) y del mercado de valores (SP500).

El insignificante peso de los otros factores incluidos en la muestra, y cuya importancia es reconocida en diferentes estudios mencionados en la Introduccion, es solo de orden estadístico y no debe mal interpretarse. La amplitud del periodo considerado disminuye, sin duda, el poder explicativo de los factores descartados porque resulta claro que su influencia es más notoria en la parte ascendente o descendente de las tendencias de la figura 1, pero no en la suma de las dos. Los cambios producidos por los movimientos de las tasas de intereses, las burbujas accionarias o los shocks macroeconomicos son, sin duda, mejor apreciadas en el corto plazo o bajo esquemas de medicion que incluyan regimenes cambiantes en periodos muestrales pequeños. De aquí que la conclusion obtenida en este trabajo deba interpretarse más bien como un resultado de largo plazo; es decir como propiedades permanentes del oro que se adicionan a aquellas resultantes de los eventos coyunturales.

Para respaldar esta conclusión el documento desarrolla pruebas de validación y desempeño (backtesting) sobre el modelo ajustado. Las primeras se enfocan en caracterizar el comportamiento de los rendimientos logarítmicos del OROSpot a traves de la correcta modelacion de dos componentes dinámicos: la media condicional (tendencia) y la volatilidad condicional. Esta caracterizacion es lograda mediante la especificacion AR(1)-GARCH(1,1) con distri- 
bución condicional t sesgada, por ser la que cumple satisfactoriamente con los supuestos de ruido blanco de los residuales estandarizados y los ajustes de las dinámicas de tendencia y volatilidad. De acuerdo con esta especificación, los rendimientos logarítmicos de USDEER y SP500 afectan de manera más significativa las fluctuaciones de los rendimientos del OROSPOT que su propio efecto autorregresivo, debido a la mayor influencia que ejercen sobre la media condicional y a sus más altos niveles de persistencia de la volatilidad.

Con las pruebas de desempeño, el documento valida que la capacidad de pronóstico fuera de la muestra del modelo seleccionado no es inferior al de los modelos competidores bajo diferentes métricas de riesgo y de error del pronóstico de la volatilidad en 100 periodos mensuales. El resultado final es que, en términos generales, no existen diferencias significativas en la capacidad predictiva de la volatilidad de OROSpot entre el AR(1)-GARCH(1,1) y el resto de especificaciones con desempeño positivo en el backtesting, con lo que el modelo ajustado cumple con el criterio $\mathrm{C} 3$.

En general podemos decir que las etapas de selección, validación y desempeño aquí realizadas para el modelo ajustado constituyen una herramienta robusta para la medición del riesgo asociado a las variaciones del precio del oro en USD. Los gestores de portafolios con exposición a las fluctuaciones de OROSpot sobre horizontes de baja frecuencia (mensual, trimestral, semestral, anual) pueden beneficiarse substancialmente del uso de la metodología desarrollada. Sobre todo porque el tratamiento multivariado de las series de residuales estandarizados que se obtienen para OROSpot, USDEER y SP500 del filtrado GARCH (ver Tabla 9) constituye una base sólida para la generación de escenarios futuros del precio del oro.

Cabe aclarar que, no obstante su utilidad, la metodologia propuesta tiene las limitaciones de concentrarse en horizontes de riesgo de corto plazo (mensual-anual) y en obviar el tratamiento conjunto de las variaciones del oro y las de los otros regresores. Por esas razones conviene sugerir, como agenda futura de investigacion, el estudio de horizontes de riesgo mas amplios, digamos multianuales, asi como el uso de meta-distribuciones basadas en cópulas multivariadas. La inclusion de horizontes mas largos demanda, sin duda, una mayor profundidad histórica en la muestra a fin de instrumentar pruebas de desempeño (backtesting) mas generales, o cambios en los componentes estructurales de las medias y varianzas condicionales que permitan incorporar los efectos del ciclo económico mundial. Con la implementacion de cópulas, la metodologia propuesta puede caracterizar aun mejor la distribución conjunta del vector aleatorio asociado a los procesos de ruido de las dinámicas de la media y varianza condicionales y, de esa manera, estudiar los comovimientos del oro y de los otros regresores en circunstancias variadas. Ademas, debido a las caracteristicas de las distribuciones de rendimientos del OROSpot y sus determinantes (con colas pesadas y asimetrícas), el estudio de cópulas puede ser un vehiculo adecuado para hacer un tratamiento detallado de la dependencia de cola en los procesos de ruido subyacentes. 


\section{Referencias}

Batten, J., Ciner, C., y Lucey, B. (2010). The macroeconomic determinants of volatility in precious metal markets. Resources Policy, 35, 65-71. https://doi.org/10.1016/j.resourpol.2009.12.002

Baur, D., Beckmann,J., y Czudaj, R. (2016). A melting pot. Gold Price forecasts under model and parameter uncertainty. International Review of Financial Analysis, 48, 282-291. https://doi.org/10.1016/j.irfa.2016.10.010

Cai, J., Cheung,Y., y Wong, M.(2001).What moves the gold market? Journal of Futures Markets 21,257-278. https://doi.org/10.1002/1096-9934(200103)21:3<257::AID-FUT4>3.0.CO;2-W

Christoffersen, P.F. (1998). Evaluating Interval Forecasts. International Economic Review, 39, 841-862. http:// dx.doi.org/10.2307/2527341

Engle, R. y V. Ng. (1993). Measuring and Testing the Impact of News on Volatility. Journal of Finance, 43, 17491778. http://dx.doi.org/10.2307/2329066

Granger, C. W. J. (1969). Investigating causal relations by econometric models and cross-spectral methods. Econometrica, 37, 424-438. http://dx.doi.org/10.2307/1912791

Hamilton, J.D. (1994). Time Series Analysis. Princeton University Press, Princeton.

Hansen, P.R. (2005). A test for superior predictive ability. Journal of Business \& Economic Statistics, 23, 365-380. http://dx.doi.org/10.1198/073500105000000063

Hansen, P. R. y Lunde, A. (2005). A forecast comparison of volatility models: Does anything beat a GARCH $(1,1)$ ? Journal of Applied Econometrics, 20, 873-889. http://dx.doi.org/10.1002/jae.800

Hong, Y. y Li, H. (2005). Nonparametric specification testing for continuous-time models with applications to term structure of interest rates. Review of Financial Studies, 18(1), 37-84. https://dx.doi.org/10.1093/rfs/hhh006

Johansen, S. (1995). Likelihood Based Inference in Cointegrated Vector Autoregressive Models. Oxford University Press, Oxford.

Kristajanpoller, W. y Minutolo, M. (2015). Gold price volatility: A forecasting approach using the artificial neural network-GARCH model. Expert systems with applications, 42,7245-7251.http://dx.doi.org/10.1016/j.eswa.2015.04.058

Kupiec, P. (1995). Techniques for Verifying the Accuracy of Risk Management Models. Journal of Derivatives, 3(2), 73-84. http://dx.doi.org/10.3905/jod.1995.407942

Lütkepohl, H. (2006). New Introduction to Multiple Time Series Analysis, Springer, New York. http://dx.doi. org/10.1007/978-3-540-27752-1

Newey, W. y West, K. (1987). A simple positive semidefinite, heteroscedasticity and autocorrelation consistent covariance matrix. Econometrica, 55, 863-898. http://dx.doi.org/10.2307/1913610

Nyblom, J. (1989). Testing for the constancy of parameters over time. Journal of the American Statistical Association, 84, 223-230. http://dx.doi.org/10.1080/01621459.1989.10478759

Pierdzioch, C., Risse, M., y Rohloff, S. (2016). A boosting approach to forecasting the volatility of gold-price fluctuations under flexible loss. Resources Policy, 47, 95-107. http://dx.doi.org/10.1016/j.resourpol.2016.01.003

Piplack, J. y Straetmans, S. (2010). Comovements of different asset classes during market stress. Pacific Economic Review, 15, 385-400. https://doi.org/10.1111/j.1468-0106.2010.00509.x

Poshakwale, S. y Mandal, A. (2016). Determinants of asymmetric return comovements of gold and other assets. International Review of Financial Analysis, 47, 229-242. https://doi.org/10.1016/j.irfa.2016.08.001

Sims, C. (1980). Macroeconomics and Reality. Econometrica 48 (1), 1-48. http://dx.doi.org/10.2307/1912017

Trück, S. y Liang, K. (2012). Modelling and forecasting volatility in the gold market. International Journal of Banking and Finance, 9 (1), 48-80. https://doi.org/10.1016/j.eswa.2015.04.058

Tully, E. y Lucey, B. (2007). A power GARCH examination of the gold market. Research in International Business and Finance, 21, 316-325. https://doi.org/10.1016/j.ribaf.2006.07.001

Vivian, A. y Wohar, M. (2012). Commodity volatility breaks. Journal of International Financial Markets, Institutions and Money, 22, 395-422. https://doi.org/10.1016/j.intfin.2011.12.003

White, H. (1980). A heteroscedasticity consistent covariance matrix estimator and a direct test for heteroscedasticity. Econometrica, 48, 827-838. http://dx.doi.org/10.2307/1912934

White, H. (1982). Maximum likelihood estimation of misspecified models. Econometrica, 50 (1), 1-25. http:// dx.doi.org/10.2307/1912526. 


\section{Anexo}

Tabla A1

Parámetros estimados del modelo VAR de la Tabla 4

\begin{tabular}{ccccc}
\hline & $\mathrm{t}$ & $\ln \left(\mathrm{OROSpot}_{\mathrm{t}-1}\right)$ & $\ln \left(\mathrm{USDEER}_{\mathrm{t}-1}\right)$ & $\ln \left(\mathrm{SP}_{\mathrm{t} 00_{\mathrm{t}-1}}\right)$ \\
\hline $\ln \left(\right.$ OROSpot $\left._{\mathrm{t}}\right)$ & 0.0002 & 0.9883 & 0.0646 & -0.0355 \\
& $(8.4 \mathrm{E}-05)$ & $(7.2 \mathrm{E}-03)$ & $(2.4 \mathrm{E}-02)$ & $(1.6 \mathrm{E}-02)$ \\
$\ln \left(\right.$ USDEER $\left._{\mathrm{t}}\right)$ & -0.0001 & & 0.9718 & 0.0177 \\
& $(2.6 \mathrm{E}-05)$ & & $(7.5 \mathrm{E}-03)$ & $(4.9 \mathrm{E}-03)$ \\
$\ln \left(\right.$ SP500 $\left._{\mathrm{t}}\right)$ & & & 0.9741 \\
& & & $(1.4 \mathrm{E}-02)$ \\
\hline
\end{tabular}

Nota: Los errores estándares se presentan entre paréntesis. Se eliminaron los coeficientes con valor $\mathrm{p}>0.05$. Si se considera un nivel de significancia del $10 \%$ resulta que $\ln ($ OROSpott-1) tiene un efecto parcial igual a 0.012 sobre $\ln$ (SP500t-1). No obstante, esto no afecta las conclusiones derivadas del ajuste del modelo VAR.

Fuente: elaboración propia.

Tabla A2

Matrices de autocorrelación cruzada de los rendimientos de OROSpot, USDEER y SP500

\begin{tabular}{|c|c|c|c|c|c|c|c|c|c|c|c|c|c|c|}
\hline \multicolumn{3}{|c|}{ Rezago 1} & \multicolumn{3}{|c|}{ Rezago 2} & \multicolumn{3}{|c|}{ Rezago 3} & \multicolumn{3}{|c|}{ Rezago 4} & \multicolumn{3}{|c|}{ Rezago 5} \\
\hline-0.10 & 0.05 & -0.06 & -0.05 & 0.02 & -0.03 & 0.02 & 0.00 & -0.02 & 0.02 & 0.00 & -0.02 & 0.01 & -0.04 & 0.00 \\
\hline-0.07 & 0.10 & 0.02 & -0.03 & 0.02 & 0.11 & -0.10 & 0.11 & -0.02 & -0.10 & 0.11 & -0.02 & -0.03 & -0.01 & -0.02 \\
\hline-0.08 & -0.04 & 0.08 & 0.07 & 0.03 & -0.03 & 0.03 & -0.06 & 0.10 & 0.03 & -0.06 & 0.10 & -0.07 & 0.02 & 0.07 \\
\hline \multicolumn{3}{|c|}{ Lag 6} & \multicolumn{3}{|c|}{ Lag 7} & \multicolumn{3}{|c|}{ Lag 8} & \multicolumn{3}{|c|}{ Lag 9} & \multicolumn{3}{|c|}{ Lag 10} \\
\hline 0.05 & 0.02 & -0.08 & 0.10 & -0.11 & -0.01 & -0.02 & -0.03 & -0.06 & -0.01 & 0.05 & -0.01 & 0.07 & -0.11 & -0.02 \\
\hline-0.06 & 0.03 & 0.03 & -0.13 & 0.11 & 0.08 & 0.11 & -0.16 & 0.10 & -0.04 & -0.04 & 0.03 & -0.12 & 0.11 & 0.01 \\
\hline \multirow[t]{4}{*}{-0.04} & 0.02 & 0.06 & 0.10 & -0.01 & -0.06 & -0.02 & 0.05 & 0.02 & -0.05 & 0.03 & 0.09 & 0.01 & 0.07 & 0.00 \\
\hline & & Rezago & 1 & 2 & 3 & 4 & 5 & 6 & 7 & 8 & 9 & 10 & & \\
\hline & & $\begin{array}{l}\text { Li Mc- } \\
\text { Leod }\end{array}$ & 21.77 & 32.70 & 40.11 & 47.08 & 53.94 & 66.72 & 83.14 & 93.06 & 107.22 & 121.61 & & \\
\hline & & valor $\mathrm{p}$ & 0.01 & 0.02 & 0.05 & 0.10 & 0.17 & 0.11 & 0.05 & 0.05 & 0.03 & 0.01 & & \\
\hline
\end{tabular}

Nota: El panel superior contiene matrices de autocorrelación cruzada para los casos de uno hasta diez rezagos. Para cada número de rezagos $1=1,2, \ldots, 10$, la entrada (i,j) de cada submatriz corresponde a la estimación muestral de la correlación lineal entre las series de rendimientos $r_{-}(i, t)$ y $r_{-}(j, t-1)$, donde los valores de los índices i,j=1,2,3 corresponden a OROSpot, USDEER y SP500, respectivamente. El panel inferior presenta el valor calculado para la estadística de prueba propuesta por Li y McLeod (1981) para contrastar la hipótesis nula de que las matrices de autocorrelación cruzada de hasta el orden de rezago considerado son idénticas a la matriz cero. Se incluye también el valor $\mathrm{p}$ correspondiente.

Fuente: elaboración propia 